\title{
Representation and Reasoning about Strategic Abilities with $\omega$-Regular Properties
}

\author{
Liping Xiong ${ }^{1, *}$ and Sumei Guo ${ }^{2, *}$ \\ 1 School of Computer, South China Normal University, Guangzhou 510631, China \\ 2 School of Computer Technology, Beijing Institute of Technology, Zhuhai, Zhuhai 519000, China \\ * Correspondence: xiongliping@scnu.edu.cn (L.X.); guo_sm@bitzh.edu.cn (S.G.)
}

check for

updates

Citation: Xiong, L.; Guo, S. Representation and Reasoning about Strategic Abilities with $\omega$-Regular Properties. Mathematics 2021, 9, 3052. https://doi.org/10.3390/math9233052

Academic Editor: Răzvan Diaconescu

Received: 22 September 2021

Accepted: 24 November 2021

Published: 27 November 2021

Publisher's Note: MDPI stays neutral with regard to jurisdictional claims in published maps and institutional affiliations.

Copyright: (c) 2021 by the authors. Licensee MDPI, Basel, Switzerland. This article is an open access article distributed under the terms and conditions of the Creative Commons Attribution (CC BY) license (https:// creativecommons.org/licenses/by/ $4.0 /)$.

\begin{abstract}
Specification and verification of coalitional strategic abilities have been an active research area in multi-agent systems, artificial intelligence, and game theory. Recently, many strategic logics, e.g., Strategy Logic (SL) and alternating-time temporal logic (ATL*), have been proposed based on classical temporal logics, e.g., linear-time temporal logic (LTL) and computational tree logic (CTL*), respectively. However, these logics cannot express general $\omega$-regular properties, the need for which are considered compelling from practical applications, especially in industry. To remedy this problem, in this paper, based on linear dynamic logic (LDL), proposed by Moshe Y. Vardi, we propose LDLbased Strategy Logic (LDL-SL). Interpreted on concurrent game structures, LDL-SL extends SL, which contains existential/universal quantification operators about regular expressions. Here we adopt a branching-time version. This logic can express general $\omega$-regular properties and describe more programmed constraints about individual/group strategies. Then we study three types of fragments (i.e., one-goal, ATL-like, star-free) of LDL-SL. Furthermore, we show that prevalent strategic logics based on LTL/CTL* , such as SL/ATL*, are exactly equivalent with those corresponding star-free strategic logics, where only star-free regular expressions are considered. Moreover, results show that reasoning complexity about the model-checking problems for these new logics, including one-goal and ATL-like fragments, is not harder than those of corresponding SL or ATL*.
\end{abstract}

Keywords: strategic abilities; $\omega$-regular properties; linear dynamic logic; strategic logics; model checking; concurrent game structure

\section{Introduction}

For the specification of ongoing behaviours of reactive systems, the use of temporal logics has become one of the significant developments in formal reasoning [1-3]. However, interpreted over Kripke structures, traditional temporal logics can only quantify the computations of the closed systems in a universal/existential manner. In order to reason in multi-agent systems, we need to specify the ongoing strategic behaviours [4].

Since Alur and Henzinger [5] proposed alternating-time temporal logic (ATL/ATL*) in 2002, strategy specification and verification has been an active research area in multiagent systems, artificial intelligence, and game theory. In recent years, there have been many extensions or variants of strategic logics proposed to reason about coalitional strategic abilities. For instance, in [6], Chatterjee et al. proposed strategy logic, which treats strategies as explicit first-order objects in turn-based games with only two agents; Mogavero et al. extended this logic with explicit strategy quantifications and agent bindings in multi-agent concurrent systems [7]; in order to reason about uniqueness of Nash Equilibria, Aminof et al. introduced a graded strategic logic [8]; in [9], Bozzelli et al. considered strategic reasoning with linear past in alternating-time temporal logic; and in [10], Belardinelli et al. studied strategic reasoning with knowledge. These logics are interpreted over concurrent game structures, in which agents act concurrently and instantaneously. Each agent acts independently and interacts with other agents. Formulas of these logics are used to specify an individual's or a group's strategic abilities. 
In ATL/ATL*, strategic abilities for coalition $A$ (i.e., a set of agents) are expressed as $\langle\langle A\rangle\rangle \psi$, representing that coalition $A$ has a group strategy to make sure that goal $\psi$ holds, no matter which strategies are chosen by other agents outside of $A$, here $\psi$ can be any temporal formula. A much more expressive strategic logic is Strategy Logic (SL) [6,7], which is a multi-agent extension of linear-time temporal logic (LTL) [11] with the concepts of agent bindings and strategy quantification. In SL, we can explicitly reason about the agent's strategy itself, allow different agents to share the same strategy, and also represent the existence of deterministic multi-player Nash equilibria.

However, on one hand, existing strategic logics are mainly based on the classical temporal logics. For instance, the underlying logics of ATL, ATL*, alternating-time mucalculus (AMC) [5], and SL are temporal logic computational tree logic CTL [12], CTL* [3], $\mu$-calculus [13], and LTL, respectively. However, they cannot express general $\omega$-regular properties, such as "property $p$ holds in any even steps in an infinite sequence, and holds in odd steps or not" [14].

On the other hand, the need of a declarative and convenient temporal logic, which can express any general $\omega$-regular expression, is considered compelling from a practical viewpoint in industry [15]. In some papers, e.g., [16], the authors introduce regular expressions or automaton directly into LTL to express $\omega$-regular properties. However, regular expressions or automaton are all too low level as a formalism for expressing temporal specifications. In 2011, Moshe Y. Vardi proposes a novel logic, named linear dynamic logic (LDL) [17], which merges LTL with regular expression in a very natural way and adopts exactly the syntax of propositional dynamic logic (PDL) [18]. LDL has three advantages:

(1) It has the same expressive power as $\omega$-regular expression, which is also equivalent with monadic second-order logic over infinite traces [19];

(2) It retains the declarative nature and intuitive appeal of LTL [20];

(3) The model checking complexity of LDL is PSPACE-complete $[17,21]$, which is the same as that of LTL.

In order to express any $\omega$-regular properties in strategic logic, in [22], Liu et al. propose a logic JAADL to specify joint abilities of coalitions, which combines alternating-time temporal logic with LDL. However, in JAADL, the authors consider a very complex semantics and study the model checking complexity with imperfect recall for JAADL.

Similarly, to remedy the inability to express any general $\omega$-regular temporal goal in strategic abilities in SL, we propose a novel strategic logic, called LDL-based Strategy Logic, abbreviated as LDL-SL. It can explicitly represent and reason about strategies and specify expressive strategic abilities for coalitions about more representative temporal goals, which can be general $\omega$-regular properties. By combining LDL and SL, LDL-SL becomes a natural and intuitive strategic logic to specify more expressive properties. (In [23], the authors propose a strategy logic based on LDL interpreted over interpreted systems with bounded private actions.)

In this paper, we show that LDL-SL is much more expressive than SL and LDL and prove that the model checking complexity of LDL-SL is nonelementary-hard [24]. Moreover, we study fragments of LDL-SL and their model-checking complexities, and we define three types of strategic logics: ATL-like, one-goal, and star-free. The former two, which are fragments for LDL-SL, have the same expressivity as those based on LTL or CTL*, and the model-checking problems are also the same. As for the last, firstly, we formally define the star-free LDL logic and prove it is equivalent with LTL. By this, we know that the corresponding star-free strategic logics are equivalent with those based on LTL/CTL*. Furthermore, the model-checking problems of these new logics, based on LDL, are the same as those based on LTL/CTL*. Furthermore, we show that the model-checking problem complexities of these logics are either 2EXPTIME-complete or nonelementary-hard.

Therefore, in any case, LDL can be viewed as a good and natural underlying temporal logic of strategic logics.

The paper is organized as follows. Section 2 introduces LDL, and its classical temporal logic fragments and then introduces the syntax and semantics of strategic logics. Section 
3 indicates that LTL is equivalent with star-free fragment of LDL. In the next section, we propose the LDL-based strategy logic (LDL-SL) and give fragments of LDL-SL. Furthermore, we present the relations for expressivity among strategic logics. Moreover, the model checking problems for these new proposed strategic logics are considered. Finally, we present conclusions and future work.

\section{Preliminaries}

In this section, firstly, we introduce temporal logics including such as $\mathrm{CDL}^{*}$ and its fragments LDL, LTL, and CTL*. Then we introduce strategic logics whose underlying logics are LTL and CTL*.

In this paper, we fix two non-empty finite sets, which are atomic proposition set $A P$, agent set $A g$, and one nonempty countable set of strategy variable Var. By $\mathcal{L}(A P)$, we denote the set of propositional formulas over $A P$. In this paper, we use true (resp. false) to refer to valid (resp. contradiction) formula.

\subsection{Temporal Logics}

Computational-tree dynamic logic $\left(\mathrm{CDL}^{*}\right)$ [25] is a branching-time extension of $\mathrm{LDL}$, which adopts the syntax from propositional dynamic logic (PDL).

Definition 1 (Syntax of CDL*). The syntax of is defined inductively by:

$$
\begin{gathered}
\text { State formula } \varphi::=p|\neg \varphi| \varphi \wedge \varphi \mid \boldsymbol{E} \psi \\
\text { Path formula } \psi::=\varphi|\neg \psi| \psi \wedge \psi \mid\langle\rho\rangle \psi \\
\text { Path expression } \rho::=\Phi|\psi ?| \rho+\rho|\rho ; \rho| \rho^{*}
\end{gathered}
$$

where, $p \in A P$, and $\Phi \in \mathcal{L}(A P)$.

Intuitively, the path formula $\langle\rho\rangle \psi$ means that from the current instant, there exists an execution satisfying the path expression $\rho$ s.t. Its last instant satisfies $\psi$, and the state formula $\mathbf{E} \psi$ means that there exists a reachable path that makes the path formula $\psi$ hold.

Let $\mathbf{A}$ define the dual of $\mathbf{E}$, i.e., $\mathbf{A}=\neg \mathbf{E} \neg$, and let $[\rho]$ define the dual of $\langle\rho\rangle$, i.e., $[\rho]=\neg\langle\rho\rangle \neg$.

LDL is a linear-time fragment of CDL*, just as LTL is a fragment of CTL* The syntax of LDL is

$$
\psi::=p|\neg \psi| \psi \wedge \psi \mid\langle\rho\rangle \psi, \quad \text { and } \rho::=\Phi|\psi ?| \rho+\rho|\rho ; \rho| \rho^{*} .
$$

Furthermore, $\mathrm{CTL}^{*}\left(\right.$ resp. LTL) is a fragment of $\mathrm{CDL}^{*}($ resp. LDL), where $\langle\rho\rangle$ is replaced by next-time $\bigcirc$, eventuality $\diamond$, and until $\mathcal{U}$, three temporal operators.

Any LTL formula can be linearly expressed in LDL, for instance, $\bigcirc p \doteq\langle$ true $\rangle$, $\diamond p \doteq\left\langle\right.$ true $\left.e^{*}\right\rangle p$, and $p \mathcal{U} q \doteq\left\langle(p ; \text { true })^{*}\right\rangle q$, when $p, q \in \mathcal{L}(A P)$.

Definition 2 (Kripke Model). A Kripke model $M$ is a tuple $(W, R, V)$, where $W$ is a finite non-empty set of possible worlds; $R \subseteq W \times W$, which is a left-total relation over $W$, i.e., for any $w \in W$, there exists $a w^{\prime} \in W$ s.t., $w R$ w' and $V: W \rightarrow 2^{A P}$ is a valuation function.

In a Kripke model $M=(W, R, V)$, by $\operatorname{Path}(w)$ we denote the set of infinite reachable sequences (i.e., path) $\pi=w_{0} w_{1} \cdots$ from $w$, where $w_{0}=w$ and $w_{i} R w_{i+1}$ for all $i \in \mathbb{N}$. Let $\pi_{i}$ denote the $i$-th element $w_{i}$ in $\pi$, and $\pi_{\geq i}$ denote the suffix of $\pi$, i.e., $\pi_{\geq i}=w_{i} w_{i+1} \cdots$, and let $\pi_{\leq i}$ denote the prefix of $\pi$, i.e., $\pi_{\leq i}=w_{0} w_{1} \cdots w_{i}$.

The semantics of $\mathrm{CDL}^{*}$ is defined inductively as follows.

Given a $\mathrm{CDL}^{*}$ state formula $\varphi$, a Kripke model $M$ and a state $w$ in $M$, the relation $M, w \models \varphi$ is defined as follows.

- $\quad M, w \models p \quad$ iff $\quad p \in V(w)$, here $p \in A P$ ； 
- $\quad M, w \mid \neg \varphi \quad$ iff $\quad M, w \not \models \neg \varphi$;

- $\quad M, w \models \varphi_{1} \wedge \varphi_{2} \quad$ iff $\quad M, w \models \varphi_{1}$ and $M, w \mid=\varphi_{2}$;

- $\quad M, w \models \mathbf{E} \psi \quad$ iff $\quad$ there exists $\pi \in \operatorname{Path}(w)$ s.t. $\pi, 0=\psi$.

Given a CDL* path formula $\psi$, a path $\pi$ in $M$, and $i \in \mathbb{N}$, the relation $\pi, i \models \psi$ is defined as follows.

- $\quad \pi, i=\varphi \quad$ iff $\quad M, \pi_{i}=\varphi$, here $\varphi$ is a $\mathrm{CDL}^{*}$ state formula;

- $\pi, i=\neg \psi$ iff $\pi, i \not \models \psi$;

- $\pi, i=\psi_{1} \wedge \psi_{2} \quad$ iff $\quad \pi, i=\psi_{1}$ and $\pi, i=\psi_{2}$;

- $\pi, i=\langle\rho\rangle \psi \quad$ iff $\quad$ there exists $j$ such that $(i, j) \in \mathcal{R}(\rho, \pi)$ and $\pi, j \models \psi$.

Given a path expression $\rho$ and a path $\pi$ in $M$, for $i, j \in \mathbb{N}$, the relation $(i, j) \in \mathcal{R}(\rho, \pi)$ is defined as follows:

- $\quad(i, j) \in \mathcal{R}(\Phi, \pi) \quad$ iff $\quad j=i+1$, and $\pi, i \models \Phi$, here $\Phi \in \mathcal{L}(A P)$;

- $\quad(i, j) \in \mathcal{R}(\psi ?, \pi) \quad$ iff $\quad j=i$, and $\pi, j \models \psi$;

- $\quad(i, j) \in \mathcal{R}\left(\rho_{1}+\rho_{2}, \pi\right) \quad$ iff $\quad(i, j) \in \mathcal{R}\left(\rho_{1}, \pi\right)$ or $(i, j) \in \mathcal{R}\left(\rho_{2}, \pi\right)$;

- $\quad(i, j) \in \mathcal{R}\left(\rho_{1} ; \rho_{2}, \pi\right) \quad$ iff there exists $k \in \mathbb{N}, i \leq k \leq j$, satisfying that $(i, k) \in$ $\mathcal{R}\left(\rho_{1}, \pi\right)$ and $(k, j) \in \mathcal{R}\left(\rho_{2}, \pi\right)$;

- $\quad(i, j) \in \mathcal{R}\left(\rho^{*}, \pi\right) \quad$ iff $\quad j=i$, or $(i, j) \in \mathcal{R}\left(\rho ; \rho^{*}, \pi\right)$.

\subsection{Strategic Logics Based on Classical Temporal Logics}

SL [24] is an expressive logic, which can explicitly reason about agents' strategies in multi-agent concurrent systems. In [26], Knight and Maubert propose a branching-time version BSL of SL, which is equivalent to SL. Here we introduce BSL with some minor changes, still equivalent with SL.

Definition 3 (BSL Formula). BSL formulas are defined inductively by:

$$
\begin{gathered}
\text { State formula } \varphi::=p|\neg \varphi| \varphi \wedge \varphi|(a, x) \varphi|\langle\langle x\rangle\rangle \varphi \mid \boldsymbol{E} \psi \\
\text { Path formula } \psi::=\varphi|\neg \psi| \psi \wedge \psi|\bigcirc \psi| \diamond \psi \mid \psi \mathcal{U} \psi,
\end{gathered}
$$

here $p \in A P, a \in A g$, and $x \in$ Var.

Syntactically, BSL extends linear-time temporal logic LTL with two operators. Intuitively, $\langle\langle x\rangle\rangle$ (resp. $(a, x))$ means "there exists a strategy $x$ " (resp. "bind agent a to the strategy associated with variable $x$ "). Here, let $\llbracket x \rrbracket=\neg\langle\langle x\rangle\rangle \neg$, which means "for all strategies $x$ ".

For a BSL formula $\varphi$, let free $(\varphi) \subseteq \operatorname{Var} \cup A g$ denote the set of free strategy variables and agents of $\varphi$. Informally, free $(\varphi)$ contains all strategy variables (resp. agents) for which there exists an agent binding but no quantifications (resp. no agent binding after the occurrence of a temporal operator). Here the formal definition refers to [24].

Since CTL* (resp. ATL*) is a fragment of ATL* [5] (resp. SL [24]), and BSL is equivalent with SL [26], then both $\mathrm{CTL}^{*}$ and $\mathrm{ATL}^{*}$ are fragments of BSL.

Now we introduce the semantics model of BSL based on the notion of concurrent game structure [5].

Definition 4 (Concurrent Game Structure). A concurrent game structure (CGS) $\mathcal{G}$ has five components $\left\langle A c t, W, \lambda, \tau, w^{0}\right\rangle$ :

- $\quad$ Act (resp. W) is a non-empty finite sets of actions (resp. states);

- $w^{0}$ is an initial state in $W$;

- $\lambda: W \rightarrow 2^{A P}$ is a valuation function;

- transition function $\tau: W \times A c t^{A g} \rightarrow W$ maps a state and a decision to next state.

$A$ decision is a function from $\mathrm{Ag}$ to $A c t$, by $A c t^{A g}$ we denote Dc.

In fact, a concurrent game structure can be viewed as a multi-player game, in which all agents strategically perform joint actions. Before defining the semantics of BSL, first we 
present relevant notations and definitions, namely track, strategy, strategy assignment, and outcome.

Definition 5 (Track). In a CGS $\mathcal{G}=\left\langle A c t, W, \lambda, \tau, w^{0}\right\rangle$, a finite state sequence $h=w_{0} w_{1} \ldots w_{k}$ is called a track in $\mathcal{G}$ if, for each $i$ with $0 \leq i<k$, there exists $d \in D$ c s.t. $w_{i+1}=\tau\left(w_{i}, d\right)$.

Given a track $h=w_{0} w_{1} \ldots w_{k}$, let $l e n(h)$ denote the length $k+1$ of $h$, and $l s t(h)$ denote the last state $w_{k}$ of $h$.

Definition 6 (Strategy). In a CGS $\mathcal{G}=\left\langle A c t, W, \lambda, \tau, w^{0}\right\rangle$, a strategy in $\mathcal{G}$ is a function mapping a track in $\mathcal{G}$ into an action.

Intuitively, a strategy of one agent can be viewed as a plan for this agent, which contains the unique choice of action for each track in $\mathcal{G}$.

For brevity, let $\operatorname{Trk}(\mathcal{G})$ (resp. $\operatorname{Str}(\mathcal{G})$ ) denote the set of all tracks (strategies) in a CGS $\mathcal{G}$, and let $\operatorname{Trk}(\mathcal{G}, w)$ denote the set of all tracks starting with $w$.

Like the definition of variable assignment in first-order logic, a partial function $\chi$ : $\operatorname{Var} \cup A g \rightarrow \operatorname{Str}(\mathcal{G})$ is called a strategy assignment or just assignment in $\mathcal{G}$, which maps a variable or an agent to a strategy. Let $A s g(\mathcal{G})$ denote the set of all strategy assignments in CGS $\mathcal{G}$. If $A g \subseteq \operatorname{dom}(\chi), \chi$ is called complete, here $\operatorname{dom}(\chi)$ is the domain of $\chi$. For each agent $a, \chi$ is called $w$-total, if $\operatorname{Track}(\mathcal{G}, w) \subseteq \operatorname{dom}(\chi(a))$. Let $\operatorname{Asg}(\mathcal{G}, w)$ denote the set of all $w$-total assignments in $\mathcal{G}$. Let $\chi[x \mapsto g]$ denote a new strategy assignment almost like $\chi$, where the only difference is that it maps $x$ into $g$.

Let $\operatorname{out}(\mathcal{G}, \chi, w)$ denote the set of outcomes (or paths) from $w$, which is determined by $\chi$. If $\mathcal{G}$ is explicit, we omit the $\mathcal{G}$ in $\operatorname{out}(\mathcal{G}, \chi, w)$.

Definition 7 (Outcome). For any $\pi=w_{0} w_{1} \ldots, \pi \in \operatorname{out}(\mathcal{G}, \chi, w)$ iff $w_{0}=w$, for any $i \in \mathbb{N}$, there exists a joint action $d$, such that $\tau\left(w_{i}, d\right)=w_{i+1}$, satisfying $d(a)=\chi(a)\left(\pi_{\leq i}\right)$ for each $a \in \operatorname{dom}(\chi) \cap A g$.

Given a collective strategy $g_{A}$ of $A$, i.e., $\left\{g_{a}: a \in A\right\}$, by out $\left(w, g_{A}\right)$ we denote the set of legal executions from $w$ where agents in $A$ perform actions according to $g_{A}$. Formally,

$$
\operatorname{out}\left(w, g_{A}\right)=\left\{\pi \mid \pi(0)=w \wedge \exists d \in \operatorname{DC} . d(A)=g_{A}\left(\pi_{\leq k}\right) \wedge \tau\left(\pi_{k}, d\right)=\pi_{k+1}, \forall k .\right\}
$$

When $\chi$ is complete and $w$-total, there exists just one path in out $(w, \chi(A g))$, which we call $(\chi, w)$-play.

Given a CGS $\mathcal{G}=\left\langle A c, W, \lambda, \tau, w^{0}\right\rangle$, a BSL state formula $\varphi$, an assignment $\chi$, and a state $w$, the relation $\mathcal{G}, \chi, w \models \varphi$ is inductively defined as follows.

- $\mathcal{G}, \chi, w \mid=p \quad$ if and only if $\quad p \in \lambda(w)$;

- $\mathcal{G}, \chi, w \mid=\neg \varphi \quad$ if and only if $\mathcal{G}, \chi, w \not \models \varphi$;

- $\quad \mathcal{G}, \chi, w \models \varphi_{1} \wedge \varphi_{2} \quad$ if and only if $\mathcal{G}, \chi, w \models \varphi_{1}$ and $\mathcal{G}, \chi, w \models \varphi_{2}$;

- $\mathcal{G}, \chi, w=(a, x) \varphi \quad$ if and only if $\mathcal{G}, \chi[x \mapsto \chi(a)], w \models \varphi$;

- $\quad \mathcal{G}, \chi, w=\langle\langle x\rangle\rangle \varphi$ if and only if there exists $g \in \operatorname{Str}(\mathcal{G})$, s.t., $\mathcal{G}, \chi[x \mapsto g], w \models \varphi$;

- $\quad \mathcal{G}, \chi, w=\mathrm{E} \psi \quad$ if and only if there exists $\pi \in \operatorname{out}(\mathcal{G}, \chi, w)$, s.t., $\mathcal{G}, \chi, \pi, 0=\psi$.

Given a path formula $\psi$ in BSL, $i \in \mathbb{N}$, and a path $\pi$, the relation $\mathcal{G}, \chi, \pi, i \models \psi$ is defined by:

- $\mathcal{G}, \chi, \pi, i=\bigcirc \psi \quad$ if and only if $\mathcal{G}, \chi, \pi, i+1=\psi$;

- $\mathcal{G}, \chi, \pi, i=\diamond \psi \quad$ if and only if $\exists j$ with $i \leq j$, satisfying that $\mathcal{G}, \chi, \pi, j \models \psi$;

- $\mathcal{G}, \chi, \pi, i=\psi_{1} \mathcal{U} \psi_{2} \quad$ if and only if $\exists j \in \mathbb{N}$ with $i \leq j$, such that for each $k, i \leq k<j$ satisfying that $\mathcal{G}, \chi, \pi, k \models \psi_{1}$, and $\mathcal{G}, \chi, \pi, j \models \psi_{2}$.

BSL state formula $\varphi$ is called a sentence if $\operatorname{free}(\varphi)=\varnothing$. Clearly, $\mathcal{G}, \chi, w \models \varphi$ does not depend on $\chi$; hence, we can omit $\chi$ without confusion. 
In order to define the syntax of BSL[1G], we introduce the notions of quantification prefix and binding prefix [24]. A sequence $\wp=\left(\left(x_{1}\right)\right)\left(\left(x_{2}\right)\right) \cdots\left(\left(x_{n}\right)\right)$ is called quantification prefix, if $\left(\left(x_{i}\right)\right) \in\left\{\left\langle\left\langle x_{i}\right\rangle\right\rangle, \llbracket x_{i} \rrbracket\right\}$ is either an existential or universal quantification. Given a fixed set of agents $A g=\left\{a_{1}, \cdots, a_{m}\right\}$, a sequence $b=\left(a_{1}, x_{1}\right), \cdots,\left(a_{m}, x_{m}\right)$ is called a binding prefix if every agent in $A g$ occurs exactly once. A combination $\wp b$ is closed if every variable occurring in $b$ occurs in some quantifier of $\wp$.

Now the syntax of one-goal fragment BSL[1G] of BSL is defined as follows.

$$
\varphi::=p|\neg \varphi| \varphi \wedge \varphi|\mathrm{E} \psi| \wp b \varphi \text {, and } \psi::=\varphi|\neg \psi| \psi \wedge \psi|\bigcirc \psi| \diamond \psi \mid \psi \mathcal{U} \psi,
$$

where $\wp b$ is a closed combination of a quantification/binding prefix [24].

ATL*, whose underlying logic is CTL*, is a fragment of BSL[1G] [24]. Its syntax is defined by $(A \subseteq A g)$

$$
\begin{gathered}
\text { State formula } \varphi::=p|\neg \varphi| \varphi \wedge \varphi \mid\langle\langle A\rangle\rangle \psi \\
\text { Path formula } \psi::=\varphi|\neg \psi| \psi \wedge \psi|\bigcirc \psi| \diamond \psi \mid \psi \mathcal{U} \psi .
\end{gathered}
$$

For details about the semantics of ATL* and BSL[1G], see [5,24].

Here, consider the semantics of the case $\varphi=\langle\langle A\rangle\rangle \psi$ : given a CGS $\mathcal{G}$ and a state $w$,

$$
\mathcal{G}, w \models\langle\langle A\rangle\rangle \psi \quad \text { iff } \quad \text { there exist } g_{A} \text {, s.t., } \forall \pi \in \text { out }\left(w, g_{A}\right), \mathcal{G}, \pi, 0=\psi \text {. }
$$

\section{Star-Free Logic of LDL}

In this section, we first define star-free logic $\mathrm{LDL}_{s f}\left(\right.$ resp. $\mathrm{CDL}_{s f}^{*}$ ) of LDL (resp. CDL*), and then show that their expressive abilities are equivalent with LTL (resp. CTL*).

We conjecture that if regular expressions are replaced by star-free regular expressions in LDL, then the expressivity of this new temporal logic is equivalent with that of LTL. In fact, in this section, we show that it is indeed true by Theorem 1.

Definition 8 (Star-free Logic $\mathrm{LDL}_{s f}$ ). The star-free logic $L D L_{s f}$ is defined inductively by:

$$
\begin{aligned}
& \qquad D L_{\text {sf }} \text { formula } \psi::=p|\neg \psi| \psi \wedge \psi \mid\langle\rho\rangle \psi \\
& \text { Star-free path expression } \rho::=\Phi|\psi ?| \rho+\rho|\rho ; \rho| \bar{\rho}
\end{aligned}
$$

where $p \in A P$ and $\Phi \in \mathcal{L}(A P)$.

Here $\bar{\rho}$ is the complement of $\rho$. In the star-free logic $\mathrm{CDL}_{s f}^{*}$ of $\mathrm{CDL}^{*}$, the path expressions in $\mathrm{CDL}^{*}$ are just replaced by star-free path expressions as follows:

$$
\text { Star-free path expression } \rho::=\Phi|\psi ?| \rho+\rho|\rho ; \rho| \bar{\rho}
$$

In a Kripke model $M$, given a path $\pi$ and path expression $\rho$, for any $i \geq j$, define

$$
(i, j) \in \mathcal{R}(\bar{\rho}, \pi) \quad \text { if and only if } \quad(i, j) \notin \mathcal{R}(\rho, \pi) .
$$

Easily, the following simple property holds.

Lemma 1. For any path $\pi$ in a Kripke model, the following holds,

$$
\mathcal{R}(\overline{\text { false }}, \pi)=\mathcal{R}\left(\text { true }^{*}, \pi\right)=\{(i, j): j \geq i, i, j \in \mathbb{N}\} .
$$

Proof. Firstly, $(i, j) \in \mathcal{R}(\overline{\text { false }}, \pi)$ iff $(i, j) \notin \mathcal{R}($ false, $\pi)$ iff $j \geq i$; secondly, $(i, j) \in \mathcal{R}\left(\right.$ true $\left.^{*}\right)$ iff $j \geq i$.

Hence, the following two equivalent results are correct. 
Corollary 1. Given an LDL formula $\psi$, the following are valid

$$
\left\langle\text { true }^{*}\right\rangle \psi \equiv\langle\overline{\text { false }}\rangle \psi \text { and }\left[\text { true }{ }^{*}\right] \psi \equiv[\overline{\text { false }}] \psi
$$

Since first-order logic (FO) over naturals has the expressive power of star-free regular expressions [27], and LTL over the naturals has precisely the expressive power of FO [28], then LTL over naturals has the same expressivity as star-free regular expression. Now we consider the relation between $\mathrm{LTL}_{\text {and }} \mathrm{LDL}_{s}$.

In fact, for each LTL formula $\psi$, we can translate it into a star-free LDL formula $S F(\psi)$ by function $S F: \mathrm{LTL} \rightarrow \mathrm{LDL}_{s f}$ as follows:

- $\quad S F(p)=p \quad S F(\neg \psi)=\neg S F(\psi)$

- $\quad S F\left(\psi \wedge \psi^{\prime}\right)=S F(\psi) \wedge S F\left(\psi^{\prime}\right)$

- $\quad S F(\bigcirc \psi)=\langle$ true $\rangle S F(\psi) \quad S F(\diamond \psi)=\langle\overline{\text { false }}\rangle S F(\psi)$

- $\quad S F\left(\psi \mathcal{U} \psi^{\prime}\right)=S F\left(\psi^{\prime}\right) \vee\langle\overline{\overline{f a l s e}} ; \neg S F(\psi)$ ?; $\overline{\text { false }} ;$ true $\rangle S F\left(\psi^{\prime}\right)$

Obviously, the function $S F$ is well-defined; i.e., for any $\psi$ in LTL, $S F(\psi) \in \mathrm{LDL}_{S f}$. Then the following result holds.

Lemma 2. In a Kripke model $M$, for any LTL formula $\psi$, a path $\pi$, and $i \in \mathbb{N}$,

$$
\pi, i \models \psi \quad \text { if and only if } \quad \pi, i \models S F(\psi) \text {. }
$$

Proof. We show this lemma inductively as follows. Here we just consider the following cases; the others are routine.

For case $\bigcirc \psi: \pi, i \models S F(\bigcirc \psi)$ iff $\pi, i \models\langle\operatorname{true}\rangle S F(\psi)$ iff $\pi, i+1 \models S F(\psi)$ iff $\pi, i+1 \models$ $\psi$ (by induction) iff $\pi, i=\bigcirc \psi$.

For case $\nabla \psi: \pi, i \models S F(\diamond \psi)$ iff $\pi, i \models\langle\overline{\text { false }}\rangle S F(\psi)$ iff $\exists j .(i, j) \in \mathcal{R}(\overline{\text { false }}, \pi)$, s.t., $\pi, j=S F(\psi)$ iff $\exists j . i \leq j$ and $\pi, j \models S F(\psi)$ (by Lemma 1) iff $\exists j . i \leq j$ and $\pi, j \models \psi$ (by induction) iff $\pi, i \models \diamond \psi$.

For case $\psi_{1} \mathcal{U} \psi_{2}$ :

$$
\begin{aligned}
& \pi, i=S F\left(\psi_{1} \mathcal{U} \psi_{2}\right) \text { iff } \\
& \pi, i=S F\left(\psi_{2}\right) \vee\left\langle\overline{\overline{\text { false }}} ; \neg S F\left(\psi_{1}\right) ? ; \overline{\text { false }} ; \operatorname{true}\right\rangle S F\left(\psi_{2}\right) \text { iff }
\end{aligned}
$$

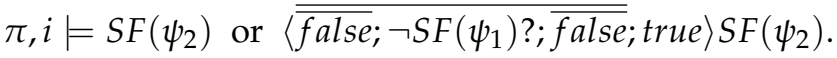

For the latter,

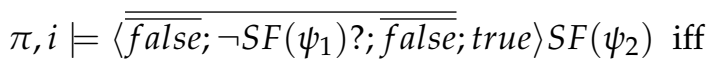

$$
\begin{aligned}
& \exists j .(i, j) \in \mathcal{R}\left(\overline{\overline{\text { false }}} ; \neg S F\left(\psi_{1}\right) \text { ?; } \overline{\text { false }} ; \text { true }, \pi\right) \text { and } \pi, j \models \psi_{2} \text { iff }
\end{aligned}
$$

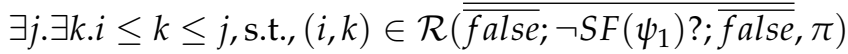

$$
\begin{aligned}
& \text { and }(k, j) \in \mathcal{R}(\text { true }, \pi) \text {, and } \pi, j=S F\left(\psi_{2}\right) \text { iff } \\
& \exists j .(i, j-1) \in \mathcal{R}\left(\overline{\overline{f a l s e}} ; \neg S F\left(\psi_{1}\right) ? ; \overline{\text { false }}, \pi\right) \text { and } \pi, j \models S F\left(\psi_{2}\right) \text {. }
\end{aligned}
$$

Then we show that

$$
\begin{aligned}
& (i, j-1) \in \mathcal{R}\left(\overline{\overline{\text { false }}} ; \neg S F\left(\psi_{1}\right) ? ; \overline{\text { false }}, \pi\right) \text { iff } \\
& (i, j-1) \notin \mathcal{R}\left(\overline{\text { false }} ; \neg S F\left(\psi_{1}\right) ? ; \overline{\text { false }}, \pi\right) \text { iff } \\
& \forall k .(i \leq k \leq j-1 \rightarrow(i, k) \notin \mathcal{R}(\overline{\text { false }}) \vee \\
& \left.(k, j-1) \notin \mathcal{R}\left(\neg S F\left(\psi_{1}\right) ? ; \overline{\text { false }}, \pi\right)\right) \text { iff } \\
& \forall k .\left(i \leq k \leq j-1 \rightarrow(k, j-1) \notin \mathcal{R}\left(\neg S F\left(\psi_{1}\right) \text { ?; } \overline{\text { false }}, \pi\right)\right) \text { iff } \\
& \forall k .\left(i \leq k \leq j-1 \rightarrow \forall k^{\prime} .\left(k \leq k^{\prime} \leq j-1 \rightarrow\left(k, k^{\prime}\right) \notin\right.\right. \\
& \left.\left.\mathcal{R}\left(\neg S F\left(\psi_{1}\right) ?, \pi\right) \vee\left(k^{\prime}, j-1\right) \notin \mathcal{R}(\overline{\text { false }}, \pi)\right)\right) \text { iff } \\
& \forall k .\left(i \leq k \leq j-1 \rightarrow \pi, k \models S F\left(\psi_{1}\right)\right) \text {. }
\end{aligned}
$$


Therefore, we have that $\pi, i=S F\left(\psi_{1} \mathcal{U} \psi_{2}\right)$ iff $\pi, i=S F\left(\psi_{2}\right)$ or there exists $j, \forall k .(i \leq$ $\left.k \leq j-1 \rightarrow \pi, k \models S F\left(\psi_{1}\right)\right)$ and $\pi, j \models S F\left(\psi_{2}\right)$ iff there exists $j$, such that for all $k . i \leq k<j$ s.t. $\pi, k \mid=\psi_{1}$ and $\pi, j=\psi_{2}$ (by induction) iff $\pi, i=\psi_{1} \mathcal{U} \psi_{2}$.

By this lemma, LTL can be linearly embeded into $\mathrm{LDL}_{s f}$. Conversely, in order to express an $\mathrm{LDL}_{s f}$ formula $\psi$ by an LTL formula, we first express $\psi$ by a first-order logic $\mathrm{FO}(\mathrm{AP})$ formula under linear order over natural numbers $\mathbb{N}$ [16]. In $\mathrm{FO}(\mathrm{AP})$, the language is formed by the binary predicate $<$, a unary predicate for each symbol in $A P$.

The first order logic $\mathrm{FO}(\mathrm{AP})$ interpretation is the form $\mathcal{I}=\left(\Delta^{\mathcal{I}},{ }^{\mathcal{I}}\right)$, where the interpretation of the following binary predicates and the constant are fixed,

- $\Delta^{\mathcal{I}}=\mathbb{N} ; \quad 0^{\mathcal{I}}=0$

- $\quad<^{\mathcal{I}}=\{(i, j): i, j \in \mathbb{N}, i<j\}$;

- $\operatorname{succ}^{\mathcal{I}}=\{(i, i+1): i \in \mathbb{N}\}$;

- $\quad{ }^{\mathcal{I}}=\{(i, i): i \in \mathbb{N}\}$.

In fact, the following properties hold.

- $\operatorname{succ}(x, y) \doteq(x<y) \wedge(\neg \exists z \cdot x<z<y)$

- $x=y \doteq \forall z . x<z \equiv y<z$

- $x \leq y \doteq x<y \vee x=y$

- $\quad 0$ can be defined as one $x$, which satisfies that $\neg \exists y \cdot \operatorname{succ}(y, x)$ or $\forall y \cdot x \leq y$.

Intuitively, $\operatorname{succ}(x, y)$ means that $y$ is an immediate successor of $x$.

Given a path $\pi$ in a Kripke model $M=(W, R, V)$, we define a corresponding first order logic interpretation $I^{\pi}$ with that for each $p \in A P$,

$$
p^{I^{\pi}}=\left\{k \mid p \in V\left(\pi_{k}\right)\right\}
$$

and interpretations of the other predicates or constant are fixed.

Now we define two functions $F O$ and $G$, which translate an $\mathrm{LDL}_{s f}$ formula into a first-order logic $\mathrm{FO}(\mathrm{AP})$ formula by induction.

- $F O(p, x)=p(x), p \in A P$;

- $\quad F O(\neg \psi, x)=\neg F O(\psi, x)$;

- $F O\left(\psi_{1} \wedge \psi_{2}, x\right)=F O\left(\psi_{1}, x\right) \wedge F O\left(\psi_{2}, x\right)$;

- $F O(\langle\rho\rangle \psi, x)=\exists y \cdot(G(\rho, x, y) \wedge F O(\psi, y))$;

- $G(\Phi, x, y)=\operatorname{succ}(x, y) \wedge F O(\Phi, x)$, here $\Phi \in \mathcal{L}(A P)$;

- $G(\psi ?, x, y)=(y=x) \wedge F O(\psi, x)$;

- $G\left(\rho_{1}+\rho_{2}, x, y\right)=G\left(\rho_{1}, x, y\right) \vee G\left(\rho_{2}, x, y\right)$;

- $G\left(\rho_{1} ; \rho_{2}, x, y\right)=\exists z .\left(x \leq z \wedge z \leq y \wedge G\left(\rho_{1}, x, z\right) \wedge G\left(\rho_{2}, z, y\right)\right)$;

- $G(\bar{\rho}, x, y)=x \leq y \wedge \neg G(\rho, x, y)$.

The function $\operatorname{FO}(\psi, x)$ and auxiliary function $G(\rho, x, y)$ are well-defined. Intuitively, here the function $G$ is used to specify the relation $\mathcal{R}(\rho, \pi)$ by formulas in FO(AP).

It is shown that the following lemma holds by induction of structures about $\mathrm{LDL}_{s} f$ formula.

Lemma 3. For any path $\pi$ in a Kripke model $M$ and $i \in \mathbb{N}$, given an $L D L_{s f}$ formula $\psi$, we have

$$
\pi, i=\psi \quad \text { if and only if } \quad I^{\pi}(i \mapsto x) \models F O(\psi, x),
$$

where $I^{\pi}$ is the corresponding first order interpretation of path $\pi$.

Proof. By induction of the formula $\mathrm{LDL}_{s f}$ formula $\psi$, we can show this lemma.

For case $\psi=p: \pi, i=p$ iff $p \in V\left(\pi_{i}\right)$ (by semantics) iff $i \in p^{\mathcal{I}^{\pi}}$ iff $I^{\pi} \models p(i)$ iff $I^{\pi}(i \mapsto x) \models F O(p, x)$ iff $I^{\pi}(i \mapsto x) \models F O(\psi, x)$;

for case $\psi=\neg \psi_{1}: \pi, i \mid=\neg \psi_{1}$ iff $\pi, i \mid=\psi_{1}$ does not hold iff $I^{\pi}(i \mapsto x) \models F O\left(\psi_{1}, x\right)$ does not hold (by induction) iff $I^{\pi}(i \mapsto x)=\neg F O\left(\psi_{1}, x\right)$ iff $I^{\pi}(i \mapsto x) \models F O\left(\neg \psi_{1}, x\right)$; 
for case $\psi=\psi_{1} \wedge \psi_{2}: \pi, i \models \psi_{1} \wedge \psi_{2}$ iff $\pi, i \models \psi_{1}$ and $\pi, i \models \psi_{2}$ iff $I^{\pi}(i \mapsto x) \models$ $F O\left(\psi_{1}, x\right)$ and $I^{\pi}(i \mapsto x) \models F O\left(\psi_{2}, x\right)$ (by induction) iff $I^{\pi}(i \mapsto x) \models F O\left(\psi_{1} \wedge \psi_{2}, x\right)$;

In order to show the case $\psi=\langle\rho\rangle \psi_{1}$, we should show the following mutually with the above (11) by induction.

$$
I^{\pi}(i \mapsto x, j \mapsto y) \models G(\rho, x, y) \quad \text { if and only if } \quad(i, j) \in \mathcal{R}(\rho, \pi) .
$$

For case $\rho=\Phi: I^{\pi}(i \mapsto x, j \mapsto y) \models G(\Phi, x, y)$ iff $I^{\pi}(i \mapsto x, j \mapsto y) \models \operatorname{succ}(x, y) \wedge$ $F O(\Phi, x)$ iff $j=i+1$ and $\pi, i=\Phi$ iff $(i, j) \in \mathcal{R}(\Phi, \pi)$.

For case $\rho=\psi$ ?: $I^{\pi}(i \mapsto x, j \mapsto y) \models G(\psi ?, x, y)$ iff $j=i$ and $I^{\pi}(i \mapsto x) \models F O(\psi, x)$ iff $j=i$ and $\pi, i \models \psi$ by induction iff $(i, j) \in \mathcal{R}(\psi$ ?,$\pi)$.

For case $\rho=\rho_{1}+\rho_{2}: I^{\pi}(i \mapsto x, j \mapsto y) \models G\left(\rho_{1}+\rho_{2}, x, y\right)$ iff $I^{\pi}(i \mapsto x, j \mapsto y) \models$ $G\left(\rho_{1}, x, y\right) \vee G\left(\rho_{2}, x, y\right)$ iff $(i, j) \in \mathcal{R}\left(\rho_{1}, \pi\right)$ or $(i, j) \in \mathcal{R}\left(\rho_{2}, \pi\right)$. The last is because by induction, we have $I^{\pi}(i \mapsto x, j \mapsto y)=G\left(\rho_{1}, x, y\right)$ iff $(i, j) \in \mathcal{R}\left(\rho_{1}, \pi\right)$, and $I^{\pi}(i \mapsto x, j \mapsto$ $y) \mid=G\left(\rho_{2}, x, y\right)$ iff $(i, j) \in \mathcal{R}\left(\rho_{2}, \pi\right)$.

For case $\rho=\rho_{1} ; \rho_{2}: I^{\pi}(i \mapsto x, j \mapsto y) \models G\left(\rho_{1} ; \rho_{2}, x, y\right)$ iff $I^{\pi}(i \mapsto x, j \mapsto y) \models$ $\exists z\left(x \leq z \wedge z \leq y \wedge G\left(\rho_{1}, x, z\right) \wedge G\left(\rho_{2}, z, y\right)\right)$ iff there exists $k$, with $i \leq k \leq j$, satisfying that $(i, k) \in \mathcal{R}\left(\rho_{1}, \pi\right)$ and $(k, j) \in \mathcal{R}\left(\rho_{2}, \pi\right)$ by induction iff $(i, j) \in \mathcal{R}\left(\rho_{1} ; \rho_{2}, \pi\right)$.

For case $\rho=\overline{\rho_{1}}: I^{\pi}(i \mapsto x, j \mapsto y) \models G\left(\overline{\rho_{1}}, x, y\right)$ iff $I^{\pi}(i \mapsto x, j \mapsto y) \models x \leq$ $y \wedge \neg G\left(\rho_{1}, x, y\right)$ iff $i \leq j$ and $I^{\pi}(i \mapsto x, j \mapsto y) \mid=\neg G\left(\rho_{1}, x, y\right)$ iff $i \leq j$ and $(i, j) \notin \mathcal{R}\left(\rho_{1}, \chi\right)$ iff $(i, j) \in \mathcal{R}\left(\overline{\rho_{1}}, \chi\right)$.

Now we show the case $\psi=\langle\rho\rangle \psi_{1}: \pi, i \models\langle\rho\rangle \psi_{1}$ iff there exists $j,(i, j) \in \mathcal{R}(\rho, \pi)$ satisfying that $\pi, j=\psi_{1}$ iff there exists $j, I^{\pi}(i \mapsto x, j \mapsto y) \models G(\rho, x, y)$ and $I^{\pi}(j \mapsto y) \models$ $F O\left(\psi_{1}, j\right)$ by induction iff $I^{\pi}(i \mapsto x) \models F O\left(\langle\rho\rangle \psi_{1}, x\right)$ by definition.

In [28], Gabbay et al. have shown that first-order logic FO for linear order over natural numbers is equivalent with LTL over infinite traces. In addition, one of the most familiar LDL formulas is [(true; true $\left.)^{*}\right] p$, which cannot be expressed in LTL [14]. Therefore, with the addition of Lemma 2 and 3, the following result holds.

Theorem 1. LTL has exactly the same expressive power as the star-free logic $L D L_{s f}$, and strictly less expressive than $L D L$.

Moreover, LTL formulas can be linearly translated into $\mathrm{LDL}_{s f}$ formulas, but the converse procedure is not. Some star-free LDL formulas are hard to encode by LTL formulas, even by LDL formulas.

\section{Strategic Logics Based on LDL/LDL $\mathrm{L}_{s}$}

In this section, we introduce two new classes of expressive strategic logics, whose underlying logic is $\mathrm{LDL}$ and $\mathrm{LDL}_{s f}$, respectively. The former can express $\omega$-regular properties, and the latter has the same expressivity as star-free regular properties. Firstly, LDL-based Strategy Logic (abbr. LDL-SL) is introduced.

\section{1. $L D L / L D L_{s f}$-Based Strategic Logics}

Definition 9 (LDL-SL Formula). LDL-SL formulas are defined inductively as follows.

$$
\begin{aligned}
& \text { State formula } \varphi::=p|\neg \varphi| \varphi \wedge \varphi|(a, x) \varphi|\langle\langle x\rangle\rangle \mid \boldsymbol{E} \psi ; \\
& \text { Path formula } \psi::=\varphi|\neg \psi| \psi \wedge \psi \mid\langle\rho\rangle \psi ; \\
& \text { Path expression } \rho::=\Phi|\psi ?| \rho+\rho|\rho ; \rho| \rho^{*},
\end{aligned}
$$

where $a \in A g, x \in$ Var, $p \in A P$, and $\Phi \in \mathcal{L}(A P)$.

In fact, LDL-SL is a logic that combines BSL with LDL. LDL-SL formula is defined recursively by three components: state formula, path formula, and path expression. Now we present the complete definition about the semantics of LDL-SL formula. 
Given a CGS $\mathcal{G}$, a state formula $\varphi$, a strategy assignment $\chi$, and a state $w$, the relation $\mathcal{G}, \chi, w \models \varphi$ is defined as follows.

- $\mathcal{G}, \chi, w=p \quad$ if and only if $\quad p \in \lambda(w)$;

- $\mathcal{G}, \chi, w \mid=\neg \varphi \quad$ if and only if $\mathcal{G}, \chi, w \not=\varphi$;

- $\quad \mathcal{G}, \chi, w=\varphi_{1} \wedge \varphi_{2} \quad$ if and only if $\mathcal{G}, \chi, w \models \varphi_{1}$ and $\mathcal{G}, \chi, w \models \varphi_{2}$;

- $\mathcal{G}, \chi, w=(a, x) \varphi \quad$ if and only if $\mathcal{G}, \chi[a \mapsto \chi(x)], w \models \varphi$;

- $\mathcal{G}, \chi, w \mid=\langle\langle x\rangle\rangle \varphi \quad$ if and only if $\exists g \in \operatorname{Str}(\mathcal{G})$ s.t., $\mathcal{G}, \chi[x \mapsto g], w \models \varphi$;

- $\mathcal{G}, \chi, w \mid=\mathbf{E} \psi \quad$ if and only if $\exists \pi \in \operatorname{out}(\mathcal{G}, \chi, w)$ s.t., $\mathcal{G}, \chi, \pi, 0=\psi$.

Given a CGS $\mathcal{G}$, a path formula $\psi$, a strategy assignment $\chi$, a path $\pi$ and some $i \in \mathbb{N}$, the relation $\mathcal{G}, \chi, \pi, i \models \psi$ is defined as follows.

- $\mathcal{G}, \chi, \pi, i=\varphi \quad$ if and only if $\mathcal{G}, \chi, w \models \varphi$, here $w=\pi_{i}$;

- $\mathcal{G}, \chi, \pi, i=\neg \psi \quad$ if and only if $\mathcal{G}, \chi, \pi, i \not \models \psi$;

- $\mathcal{G}, \chi, \pi, i=\psi_{1} \wedge \psi_{2} \quad$ if and only if $\mathcal{G}, \chi, \pi, i=\psi_{1}$ and $\mathcal{G}, \chi, \pi, i=\psi_{2}$;

- $\mathcal{G}, \chi, \pi, i=\langle\rho\rangle \psi$ if and only if $\exists j .(i, j) \in \mathcal{R}(\mathcal{G}, \rho, \pi, \chi)$ and $\mathcal{G}, \chi, \pi, j \models \psi$.

The relation $(i, j) \in \mathcal{R}(\mathcal{G}, \rho, \pi, \chi)$ is defined as follows:

- $\quad(i, j) \in \mathcal{R}(\mathcal{G}, \Phi, \pi, \chi)$ if and only if $\quad j=i+1$ and $\mathcal{G}, \chi, \pi, i=\Phi$;

- $\quad(i, j) \in \mathcal{R}(\mathcal{G}, \psi ?, \pi, \chi)$ if and only if $j=i$ and $\mathcal{G}, \chi, \pi, j \models \psi$;

- $\quad(i, j) \in R\left(\mathcal{G}, \rho_{1}+\rho_{2}, \pi, \chi\right)$ if and only if $(i, j) \in \mathcal{R}\left(\mathcal{G}, \rho_{1}, \pi, \chi\right)$ or $\mathcal{R}\left(\mathcal{G}, \rho_{2}, \pi, \chi\right)$;

- $\quad(i, j) \in \mathcal{R}\left(\mathcal{G}, \rho_{1} ; \rho_{2}, \pi, \chi\right)$ if and only if there exists $k, i \leq k \leq j$, satisfying $(i, k) \in$ $\mathcal{R}\left(\mathcal{G}, \rho_{1}, \pi, \chi\right)$ and $(k, j) \in \mathcal{R}\left(\mathcal{G}, \rho_{2}, \pi, \chi\right)$;

- $\quad(i, j) \in \mathcal{R}\left(\mathcal{G}, \rho^{*}, \pi, \chi\right)$ if and only if $j=i$, or $(i, j) \in \mathcal{R}\left(\mathcal{G}, \rho ; \rho^{*}, \pi, \chi\right)$.

In the above, we omit $\mathcal{G}$ in $\mathcal{R}(\mathcal{G}, \rho, \pi, \chi)$ when there is no confusion. Intuitively, $(i, j) \in$ $\mathcal{R}(\mathcal{G}, \rho, \pi, \chi)$ means that the sequence $\pi_{i} \ldots \pi_{j}$ is a legal execution of $\rho$ under assignment $\chi$ in CGS $\mathcal{G}$.

For two special path expressions, $\psi$ ?; true and its nondeterministic iteration $(\psi \text { ?; true })^{*}$, the following properties hold, where $\psi$ is an LDL-SL path formula.

Lemma 4. Given a CGS $\mathcal{G}$, a path formula $\psi$, a path $\pi$, a strategy assignment $\chi$, and $i, j \in \mathbb{N}$,

$$
(i, j) \in \mathcal{R}(\mathcal{G}, \psi ? ; \text { true }, \pi, \chi) \quad \text { if and only if } j=i+1 \text { and } \mathcal{G}, \chi, \pi, i \models \psi \text {. }
$$

Proof. $(i, j) \in \mathcal{R}(\mathcal{G}, \psi$ ?; true, $\pi, \chi)$ iff there exists $k$ with $i \leq k \leq j$ such that $(i, k) \in$ $\mathcal{R}(\mathcal{G}, \psi ?, \pi, \chi)$ and $(k, j) \in \mathcal{R}(\mathcal{G}$, true $, \pi, \chi)$ iff there exists $k$ with $i \leq k \leq j$ such that $k=i$ and $\mathcal{G}, \chi, \pi, k=\psi$ and $j=k+1$ iff $j=i+1$ and $\mathcal{G}, \chi, \pi, i=\psi$.

Corollary 2. Given a CGS $\mathcal{G}$, a path formula $\psi$, a path $\pi$, a strategy assignment $\chi$, and $i, j \in \mathbb{N}$,

$$
(i, j) \in \mathcal{R}\left(\mathcal{G},(\psi ? ; \text { true })^{*}, \pi, \chi\right) \text { if and only if } j=i \text { or }(\forall k . i \leq k<j, \mathcal{G}, \chi, \pi, k \models \psi) \text {. }
$$

Proof. $(i, j) \in \mathcal{R}\left(\mathcal{G},(\psi ? ; \text { true })^{*}, \pi, \chi\right)$ iff $j=i$ or there exists $k(i \leq k \leq j)$ s.t. $(i, k) \in$ $\mathcal{R}(\mathcal{G},(\psi ? ;$ true $), \pi, \chi)$ and $(k, j) \in \mathcal{R}\left(\mathcal{G},(\psi ? ; \text { true })^{*}, \pi, \chi\right)$ iff $j=i$ or $(\mathcal{G}, \chi, \pi, i \models \psi$ and $\left.(i+1, j) \in \mathcal{R}\left(\mathcal{G},(\psi ? ; \text { true })^{*}, \pi, \chi\right)\right)$ by Lemma 4 iff $j=i$ or $(\mathcal{G}, \chi, \pi, i=\psi, \mathcal{G}, \chi, \pi, i+1 \models \psi$ and $\left.(i+2, j) \in \mathcal{R}\left(\mathcal{G},(\psi ? ; \text { true })^{*}, \pi, \chi\right)\right)$ iff $j=i$ or $(\mathcal{G}, \chi, \pi, i \models \psi, \mathcal{G}, \chi, \pi, i+1 \models \psi, \ldots$, and $\left.(j-1, j) \in \mathcal{R}\left(\mathcal{G},(\psi ? ; \text { true })^{*}, \pi, \chi\right)\right)$ iff $j=i$ or $(\mathcal{G}, \chi, \pi, i \models \psi, \mathcal{G}, \chi, \pi, i+1 \models \psi, \ldots$, and $\mathcal{G}, \chi, \pi, j-1 \mid=\psi$ ) repeatedly iff $j=i$ or $(\forall k . i \leq k<j, \mathcal{G}, \chi, \pi, k \mid=\psi)$.

Secondly, $\mathrm{LDL}_{s f}$-based Strategy Logic (abbr. LDL-SL ${ }_{s f}$ is introduced).

Definition 10 (LDL-SL $_{s f}$ Formula). The LDL-SL $f$ formulas are defined as follows:

$$
\begin{gathered}
\text { State formula } \varphi::=p|\neg \varphi| \varphi \wedge \varphi|\langle\langle x\rangle\rangle \varphi|(a, x) \varphi \mid \boldsymbol{E} \psi \\
\text { Path formula } \psi::=\varphi|\neg \psi| \psi \wedge \psi \mid\langle\rho\rangle \psi
\end{gathered}
$$




$$
\text { Star-free path expression } \rho:=\Phi|\psi ?| \rho+\rho|\rho ; \rho| \bar{\rho}
$$

where $a \in A g, x \in$ Var, $p \in A P$, and $\Phi \in \mathcal{L}(A P)$.

For the semantics of star-free fragment, given a CGS $\mathcal{G}$, a star-free path expression $\rho$, and a strategy assignment $\chi$, for any $i \leq j$,

$$
(i, j) \in \mathcal{R}(\mathcal{G}, \bar{\rho}, \pi, \chi) \quad \text { if and only if } \quad(i, j) \notin \mathcal{R}(\mathcal{G}, \rho, \pi, \chi) .
$$

\subsection{Fragments of LDL-SL and $L D L-S L_{s f}$}

In this subsection, we consider fragments for both LDL-SL and LDL-SL ${ }_{s f}$, including SL-like, one-goal fragments, and ATL-like fragments.

Firstly, we consider the SL-like fragment BSL of LDL-SL.

Since LTL is a sublogic of LDL, then by Corollary 2 it is easily shown that BSL is a fragment of LDL-SL by induction and semantics definition. In the following, suppose a $\operatorname{logic} \mathcal{L} \in\left\{\mathrm{BSL}, \mathrm{LDL}-\mathrm{SL}, \mathrm{ATL}^{*}, \mathrm{ADL}^{*}\right\}$, let $\mathcal{L}^{s}$ (resp, $\mathcal{L}^{p}$ ) denote all the set of state (resp. path) formulas in $\mathcal{L}$.

Theorem 2. LDL-SL is strictly more expressive than BSL.

Proof. Firstly, we define two functions $T^{s}: B S L^{s} \rightarrow L D L-S L^{s}$ and $T^{p}: B S L^{p} \rightarrow L D L-$ $S L^{p}$ by induction of structures of state formulas and path formulas.

- $\quad T^{\mathcal{S}}(p)=p ; T^{\mathcal{S}}(\neg \varphi)=\neg T^{\mathcal{S}}(\varphi) ; T^{\mathcal{S}}\left(\varphi_{1} \wedge \varphi_{2}\right)=T^{\mathcal{S}}\left(\varphi_{1}\right) \wedge T^{\mathcal{S}}\left(\varphi_{2}\right)$;

- $\quad T^{s}((a, x) \varphi)=(a, x) T^{s}(\varphi) ; T^{s}(\langle\langle x\rangle\rangle)=\langle\langle x\rangle\rangle T^{s}(\varphi) ; T^{s}(\mathbf{E} \psi)=\mathbf{E}\left(T^{p}(\psi)\right)$.

- $\quad T^{p}(\varphi)=T^{s}(\varphi) ; T^{p}(\neg \psi)=\neg T^{p}(\psi) ; T^{p}\left(\psi_{1} \wedge \psi_{2}\right)=T^{p}\left(\psi_{1}\right) \wedge T^{p}\left(\psi_{2}\right) ;$

- $\quad T^{p}(\bigcirc \psi)=\langle$ true $\rangle T^{p}(\psi) ; T^{p}(\diamond \psi)=\left\langle\right.$ true $\left.^{*}\right\rangle T^{p}(\psi) ;$

- $\quad T^{p}\left(\psi_{1} \mathcal{U} \psi_{2}\right)=\left\langle\left(T^{p}\left(\psi_{1}\right) \text { ?; true }\right)^{*}\right\rangle T^{p}\left(\psi_{2}\right)$.

By induction, both $T^{s}$ and $T^{p}$ are well-defined; i.e., for any $\varphi \in \mathrm{BSL}^{s}$ and $\psi \in \mathrm{BSL}^{p}$, $T^{\mathcal{S}}(\varphi) \in \mathrm{LDL}^{-S L}{ }^{s}$ and $T^{p}(\psi) \in \mathrm{LDL}^{-S L}{ }^{p}$.

Moreover, for any CGS $\mathcal{G}$, a BSL state formula $\varphi$, a strategy assignment $\chi$, and a state $w$, the following holds:

$$
\mathcal{G}, \chi, w \models \varphi \quad \text { if and only if } \mathcal{G}, \chi, w \models T^{\mathcal{S}}(\varphi) .
$$

For any CGS $\mathcal{G}$, a BSL path formula $\psi$, a strategy assignment $\chi$, a path $\pi$, and some $i \in \mathbb{N}$, the following holds:

$$
\mathcal{G}, \chi, \pi, i=\psi \quad \text { if and only if } \quad \mathcal{G}, \chi, \pi, i \models T^{p}(\psi) .
$$

We can show the above two mutually by induction.

It is easy to see that for the Boolean cases, the above two are obvious.

For case $\varphi=(a, x) \varphi^{\prime}: \mathcal{G}, \chi, w \models T^{S}\left((a, x) \varphi^{\prime}\right)$ iff $\mathcal{G}, \chi, w \models(a, x) T^{S}\left(\varphi^{\prime}\right)$ by definition of $T^{\mathcal{S}}$ iff $\mathcal{G}, \chi[a \mapsto \chi(x)], w \models T^{\mathcal{S}}\left(\varphi^{\prime}\right)$ by semantics definition iff $\mathcal{G}, \chi[a \mapsto \chi(x)], w \models \varphi^{\prime}$ by induction iff $\mathcal{G}, \chi, w \models(a, x) \varphi^{\prime}$ by semantics definition.

For case $\varphi=\langle\langle x\rangle\rangle \varphi^{\prime}: \mathcal{G}, \chi, w=T^{\mathcal{S}}\left(\langle\langle x\rangle\rangle \varphi^{\prime}\right)$ iff $\mathcal{G}, \chi, w=\langle\langle x\rangle\rangle T^{\mathcal{S}}\left(\varphi^{\prime}\right)$ by definition of $T^{S}$ iff $\exists g \in \operatorname{Str}(\mathcal{G}), \mathcal{G}, \chi[x \mapsto g], w \models T^{S}\left(\varphi^{\prime}\right)$ iff $\exists g \in \operatorname{Str}(\mathcal{G}), \mathcal{G}, \chi[x \mapsto g], w \models \varphi^{\prime}$ iff $\mathcal{G}, \chi, w \models\langle\langle x\rangle\rangle \varphi^{\prime}$.

For case $\varphi=\mathbf{E} \psi: \mathcal{G}, \chi, w \models T^{\mathcal{S}}(\mathbf{E} \psi)$ iff $\mathcal{G}, \chi, w \models \mathbf{E}\left(T^{p}(\psi)\right)$ by definition of $T^{\mathcal{S}}$ iff $\exists \pi \in \operatorname{out}(\mathcal{G}, \chi, w)$ s.t. $\mathcal{G}, \chi, \pi, 0 \models T^{p}(\psi)$ iff $\exists \pi \in \operatorname{out}(\mathcal{G}, \chi, w)$ s.t. $\mathcal{G}, \chi, \pi, 0 \models \psi$ iff $\mathcal{G}, \chi, w \mid=\mathbf{E} \psi$.

For case $\psi=\varphi \in \mathrm{BSL}^{s}: \mathcal{G}, \chi, \pi, i \models T^{p}(\varphi)$ iff $\mathcal{G}, \chi, \pi, i \models T^{s}(\varphi)$ by definition of $T^{p}$ iff $\mathcal{G}, \chi, \pi_{i}=T^{S}(\varphi)$ iff $\mathcal{G}, \chi, \pi(i) \models \varphi$ iff $\mathcal{G}, \chi, \pi, i=\varphi$.

For case $\psi=\bigcirc \psi^{\prime}: \mathcal{G}, \chi, \pi, i=T^{p}\left(\bigcirc \psi^{\prime}\right)$ iff $\mathcal{G}, \chi, \pi, i=\langle$ true $\rangle T^{p}\left(\psi^{\prime}\right)$ by definition of $T^{p}$ iff $\mathcal{G}, \chi, \pi, i+1 \models T^{p}\left(\psi^{\prime}\right)$ iff $\mathcal{G}, \chi, \pi, i+1 \models \psi^{\prime}$ iff $\mathcal{G}, \chi, \pi, i=\bigcirc \psi^{\prime}$. 
For case $\psi=\diamond \psi^{\prime}: \mathcal{G}, \chi, \pi, i=T^{p}\left(\diamond \psi^{\prime}\right)$ iff $\mathcal{G}, \chi, \pi, i=\left\langle\operatorname{tru} e^{*}\right\rangle T^{p}\left(\psi^{\prime}\right)$ by definition of $T^{p}$ iff there exists $j \geq i, \mathcal{G}, \chi, \pi, j=T^{p}\left(\psi^{\prime}\right)$ iff there exists $j \geq i, \mathcal{G}, \chi, \pi, j \models \psi^{\prime}$ iff $\mathcal{G}, \chi, \pi, i=\diamond \psi^{\prime}$.

For case $\psi=\psi_{1} \mathcal{U} \psi_{2}: \mathcal{G}, \chi, \pi, i=T^{p}\left(\psi_{1} \mathcal{U} \psi_{2}\right)$ iff $\mathcal{G}, \chi, \pi, i=\left\langle\left(T^{p}\left(\psi_{1}\right) \text { ?; true }\right)^{*}\right\rangle T^{p}\left(\psi_{2}\right)$ by definition of $T^{p}$ iff there exists $j$ with $(i, j) \in \mathcal{R}\left(\mathcal{G},\left(T^{p}\left(\psi_{1}\right) \text { ?; true }\right)^{*}, \pi, \chi\right)$, such that $\mathcal{G}, \chi, \pi, i=T^{p}\left(\psi_{2}\right)$ iff there exists $j$ with $j=i$ or $(\forall k, i \leq k<j$, satisfying that $\mathcal{G}, \chi, \pi, k \models$ $\left.T^{p}\left(\psi_{1}\right)\right)$, such that $\mathcal{G}, \chi, \pi, i \models T^{p}\left(\psi_{2}\right)$ by semantics definition and Corollary 2 iff there exists $j$ with $j=i$ or $\left(\forall k, i \leq k<j\right.$, satisfying that $\left.\mathcal{G}, \chi, \pi, k=\psi_{1}\right)$, such that $\mathcal{G}, \chi, \pi, i=\psi_{2}$ by induction iff $\mathcal{G}, \chi, \pi, i=\psi_{1} \mathcal{U} \psi_{2}$.

Secondly, according to a well-known property even $(q)$ "a proposition $q$ has to be true in each even state of one sequence" cannot be expressed in LTL [14], which can be expressed in LDL by $\left[(\text { true; true })^{*}\right] q$. Considering those CGSs with only one agent, LDL-SL formula $\langle\langle x\rangle\rangle(a, x) \mathbf{E}\left[(\text { true; true })^{*}\right] q$ cannot be expressed by any BSL formula.

Hence we have shown that LDL-SL is more expressively than BSL.

Secondly, we consider a one-goal fragment LDL-SL[1G] and an ATL-like fragment $\mathrm{ADL}^{*}$ of LDL-SL.

The syntax of LDL-SL[1G] is the same as that of LDL-SL, except for state formulas:

$$
\text { State formula } \varphi::=p|\neg \varphi| \varphi \wedge \varphi|\mathbf{E} \psi| \wp b \varphi \varphi,
$$

where $p \in A P$, and $\wp b$ is a closed combination of a quantification/binding prefix.

The following is ATL-like fragment ADL* of LDL-SL, of which the path formulas are different from those of ATL*.

Definition 11 (ADL* Syntax). The syntax of $A D L^{*}$ is defined as follows:

$$
\begin{gathered}
\text { State formula } \varphi::=p|\neg \varphi| \varphi \wedge \varphi \mid\langle\langle A\rangle \psi \\
\text { Path formula } \psi::=\varphi|\neg \psi| \psi \wedge \psi \mid\langle\rho\rangle \psi \\
\text { Regular expression } \rho::=\Phi|\psi ?| \rho+\rho|\rho ; \rho| \rho^{*},
\end{gathered}
$$

where $p \in A P, A \subseteq A g$, and $\Phi \in \mathcal{L}(A P)$.

By the following lemma, any ATL* formula can be expressed in $\mathrm{ADL}^{*}$.

Lemma 5. Any $A T L^{*}$ formula can be linearly encoded by one $A D L^{*}$ formula.

Proof. Define two translation functions $T^{s}: \mathrm{ATL}^{* s} \rightarrow \mathrm{ADL}^{* s}, \mathrm{~T}^{p}: \mathrm{ATL}^{* p} \rightarrow \mathrm{ADL}^{* p}$ :

- $\quad T^{s}(p)=p ; T^{s}(\neg \varphi)=\neg T^{s}(\varphi) ; T^{s}\left(\varphi_{1} \wedge \varphi_{2}\right)=T^{s}\left(\varphi_{1}\right) \wedge^{s}\left(\varphi_{2}\right) ;$

- $\quad T^{s}(\langle\langle A\rangle\rangle \psi)=\langle\langle A\rangle\rangle T^{p}(\psi)$.

- $\quad T^{p}(\varphi)=T^{s}(\varphi) ; T^{p}(\neg \psi)=\neg T^{p}(\psi) ; T^{p}\left(\psi_{1} \wedge \psi_{2}\right)=T^{p}\left(\psi_{1}\right) \wedge T^{p}\left(\psi_{2}\right) ;$

- $\quad T^{p}(\bigcirc \psi)=\langle$ true $\rangle T^{p}(\psi) ; T^{p}(\diamond \psi)=\left\langle\right.$ true $\left.^{*}\right\rangle T^{p}(\psi) ;$

- $\quad T^{p}\left(\psi_{1} \mathcal{U} \psi_{2}\right)=\left\langle\left(T^{p}\left(\psi_{1}\right) \text { ?; true }\right)^{*}\right\rangle T^{p}\left(\psi_{2}\right)$.

Here to show this lemma, similarly with those in Theorem 2, the only different case is $\varphi=\langle\langle A\rangle\rangle \psi$. Given a CGS $\mathcal{G}$, a state $w$, a state formula $\varphi$,

- $\quad$ for the case $\varphi=\langle\langle A\rangle\rangle \psi: \mathcal{G}, w=T^{s}(\langle\langle A\rangle\rangle \psi)$ iff $\mathcal{G}, w \mid=\langle\langle A\rangle\rangle T^{p}(\psi)$ by definition of $T^{s}$ iff there exist collective strategies $g_{A}$ s.t. for each $\pi \in \operatorname{out}\left(w, g_{A}\right), \mathcal{G}, \pi, 0=T^{p}(\psi)$ by semantics iff there exist collective strategies $g_{A}$ s.t. for each $\pi \in \operatorname{out}\left(w, g_{A}\right)$, $\mathcal{G}, \pi, 0=\psi$ by induction iff $\mathcal{G}, w \models\langle\langle A\rangle\rangle \psi$ by semantics.

Obviously, for any $\varphi \in \mathrm{ATL}^{* S}$, the size of $T^{S}(\varphi)$ is $O(|\varphi|)$. 
Thirdly, we consider one-goal fragment LDL-SL[1G $]_{s f}$ and ATL-like fragment ADL sf $^{*}$ of LDL-SL ${ }_{s f}$. The syntax of LDL-SL[1G] $]_{s f}$ is the same as that of LDL-SL[1G] except for regular expressions:

$$
\text { Regular expression } \rho::=\Phi|\psi ?| \rho+\rho|\rho ; \rho| \bar{\rho},
$$

where $\Phi \in \mathcal{L}(A P)$, and $\psi$ is a path formula in LDL-SL[1G $]_{s}$.

The syntax of $\mathrm{ADL}_{s f}^{*}$ is the same that of $\mathrm{ADL}^{*}$ except for regular expressions,

$$
\text { Regular expression } \rho::=\Phi|\psi ?| \rho+\rho|\rho ; \rho| \bar{\rho},
$$

where $\Phi \in \mathcal{L}(A P)$ and $\psi$ is a path formula in $\mathrm{ADL}_{s f}^{*}$.

Here we consider three kinds of fragments of LDL-SL: one-goal fragment, star-free, and ATL-like. The semantics of these logics are the same as those of LDL-SL and LDL-SL ${ }_{s f}$, respectively.

\section{Expressivity Relations among Fragments of LDL-SL and LDL-SL $s f$}

In this section, we study the expressivity relations among mentioned fragments of LDL-SL and LDL-SL $s f$. Firstly, we give the following definitions about the expressive power between two logics.

Logic $L_{1}$ is at least as expressive as logic $L_{2}$, denoted as $L_{2} \subseteq L_{1}$, if given a model $M$, for any formula $\varphi$ in $L_{2}$, there exists a formula $\varphi^{\prime}$ in $L_{1}$, satisfying that $M=\varphi$ iff $M=\varphi^{\prime}$. $L_{1}$ is strictly more expressive than $L_{2}$, denoted as $L_{2} \subsetneq L_{1}$, if $L_{2} \subseteq L_{1}$, but $L_{1} \subseteq L_{2}$ does not hold. $L_{1}$ has the same expressive power as $L_{2}$, denoted as $L_{1} \equiv L_{2}$, if $L_{1} \subseteq L_{2}$ and $L_{2} \subseteq L_{1}$. $L_{1}$ and $L_{2}$ are incomparable if neither $L_{2} \subseteq L_{1}$ nor $L_{1} \subseteq L_{2}$.

According to Theorem 1, star-free type strategic logics have the same expressive power as their corresponding strategic logics based on LTL or CTL*.

Theorem 3. Star-free strategic logics have the same expressive power as their corresponding strategic logics whose underlying logic is LTL or ATL*.

1. $A D L_{s f}^{*} \equiv A T L^{*}$;

2. $L D L-S L_{s f} \equiv B S L$;

3. $L D L-S L[1 G]_{s f} \equiv B S L[1 G]$.

Proof. By applying Lemma 2 that $\mathrm{LDL}_{s f}$ is equivalent with $\mathrm{LTL}$, these results can be shown by induction of the structures of formulas similarly. Here, we just sketch the ideas of proofs as follows.

In order to show that $\mathrm{ADL}_{s f}^{*} \subseteq \mathrm{ATL}^{*}$, by induction hypothesis, we just consider the case $\varphi=\langle\langle A\rangle\rangle \psi$, which is an $\mathrm{ADL}_{s f}^{*}$ formula. Suppose for each maximal state subformulas $\varphi^{\prime}$ in $\varphi$, by induction, there is an $\mathrm{ATL}^{*}$ formula equivalent with $\varphi^{\prime}$. If we use a new atom $p_{\varphi^{\prime}}$ to replace it, then make $\psi$ be equivalent with a pure LDL formula. By Lemma 2, replace $\psi$ with one LTL formula; and further replace those new atoms $p_{\varphi^{\prime}}$ with original ATL* state formulas. Hence the resulting formula is an ATL* state formula, equivalent with $\varphi$.

Similarly, we can show that LDL-SL ${ }_{s f} \subseteq$ BSL and LDL-SL[1G] $]_{s f} \subseteq$ BSL[1G].

For item 1: In order to show $\mathrm{ATL}^{*} \subseteq \mathrm{ADL}_{s f}^{*}$, define two functions $T^{s}$ and $T^{p}$ similarly with those in Lemma 5 except the following two cases in $T^{p}$.

$$
T^{p}(\diamond \psi)=\langle\overline{\text { false }}\rangle T^{p}(\psi), T^{p}\left(\psi_{1} \mathcal{U} \psi_{2}\right)=T^{p}\left(\psi_{2}\right) \vee\left\langle\overline{\overline{f a l s e}} ; \neg T^{p}\left(\psi_{1}\right) ? ; \overline{\text { false }} ; \text { true }\right\rangle T^{p}\left(\psi_{2}\right)
$$

Here, the proof for case $\psi=\diamond \psi_{1}$ or $\psi=\psi_{1} \mathcal{U} \psi_{2}$ about $\mathcal{G}, \pi, i \models T^{p}(\psi)$ iff $\mathcal{G}, \pi, i=\psi$ is the same as that of Lemma 2.

Similarly to Item 1 , and by Theorem 2, BSL $\subseteq \mathrm{LDL}-S L_{s f}$ and BSL[1G] $\subseteq \mathrm{LDL}-\mathrm{SL}[1 \mathrm{G}]_{s f}$ can be shown. 
Theorem 4. The following fragments are incomparable.

1. BSL and LDL-SL[1G] are incomparable.

2. $B S L[1 G]$ and $A D L^{*}$ are incomparable.

Proof. Here, we just sketch the ideas of proofs.

For item (1), we consider the following formulas:

- $\varphi_{1}=\langle\langle x\rangle\rangle \llbracket y \rrbracket\langle\langle z\rangle\rangle((a, x)(b, y) \mathbf{E} \bigcirc p \vee(a, y)(b, z) \mathbf{E} \bigcirc \neg p)$

- $\varphi_{2}=\langle\langle x\rangle\rangle(a, x)\left(\mathbf{E}\left[(\text { true; true })^{*}\right] p\right)$

where $\varphi_{1}$ is a BSL formula, but it cannot be expressed in LDL-SL[1G]; = conversely, $\varphi_{2}$ is a LDL-SL[1G] formula, but it cannot be expressed in BSL.

In order to show that $\varphi_{2}$ cannot be expressed in BSL, we consider all the CGSs with just one agent and an action. So in these CGSs, each BSL sentence is equivalent with one $\mathrm{CTL}^{*}$ state formula. Suppose $\varphi$ is a CTL* state formula with $m \bigcirc$ temporal operators; then, consider the following two CGSs with just one agent and an action-see Figure 1. In $\mathcal{G}_{1}$, $p$ holds in all states, and in $\mathcal{G}_{2}, p$ does not hold only in state $w_{2 m+1}$. Due to unique path starting from the initial state, we can see that $\varphi$ is equivalent with an LTL formula $\psi$ under each $\mathcal{G}_{i}, i \in\{1,2\}$. Then by the following theorem given by Wolper,

Theorem 4.1 ([14]) Given an atomic proposition $q$, any LTL formula $f(q)$ containing $m$

$\bigcirc$ temporal operators has the same truth value on all sequences such as $q^{k}(\neg q) q^{\omega}$, here

$k>m$ and $f(q)$ is a LTL formula containing only atomic $q$.

It holds that $\mathcal{G}_{1} \models \psi$ iff $\mathcal{G}_{2} \models \psi$. However, $\mathcal{G}_{1} \models \varphi_{2}$, but $\mathcal{G}_{2} \not \models \varphi_{2}$. Therefore, $\varphi_{2}$ cannot be expressed in BSL.

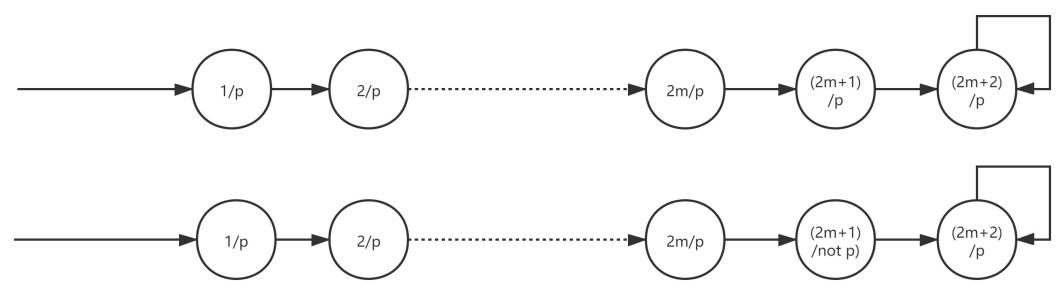

Figure 1. Two CGSs for $\varphi_{2}$ : the top is $\mathcal{G}_{1}$ and the bottom is $\mathcal{G}_{2}$.

For item (2), we consider the following two formulas:

- $\varphi_{3}=\llbracket x \rrbracket\langle\langle y\rangle\rangle[[z]](a, x)(b, y)(c, z) \mathbf{E} \bigcirc p$

- $\left.\varphi_{4}=\langle\langle\{a\}\rangle\rangle\left([\text { (rue; } \text { true })^{*}\right] p\right)$

Here, $\varphi_{3}$ is a BSL[1G] formula, but it cannot be expressed in $\mathrm{ADL}^{*}$; conversely, $\varphi_{4}$ is a $\mathrm{ADL}^{*}$ formula, but cannot be expressed in BSL[1G].

Like in [24], consider two concurrent game structures CGSs with $A P=\{p\}$ and $A g=\{a, b, c\}, \mathcal{G}_{1}=\left\langle A c_{1}, W_{1}, \lambda_{1}, \tau_{1}, w_{0}\right\rangle$, and $\mathcal{G}_{2}=\left\langle A c_{2}, W_{2}, \lambda_{2}, \tau_{2}, w_{0}\right\rangle$, where $A c_{1}=$ $\{0,1\}, A c_{2}=\{0,1,2\}, W_{1}=W_{2}=\left\{w_{0}, w_{1}, w_{2}\right\}, \lambda_{1}=\lambda_{2}$, and $D_{1}=\{00 *, 11 *\}, D_{2}=$ $\{211,202,200,00 *, 11 *, 12 *\} . \lambda_{1}\left(w_{0}\right)=\lambda_{1}\left(w_{2}\right)=\varnothing, \lambda_{1}\left(w_{1}\right)=\{p\} . \forall d \in D_{i}, \tau_{i}\left(w_{0}, d\right)=$ $w_{1} ; \forall d \in D c_{i} \backslash D_{i}, \tau\left(w_{0}, d\right)=w_{2} ; \forall d \in D c_{i}, w \in\left\{w_{1}, w_{2}\right\}, \tau_{i}(w, d)=w$, here $i \in\{1,2\}$ and $D c_{i}=A c_{i}^{A g}$. We can show that $\mathcal{G}_{1}=\varphi_{3}$, but $\mathcal{G}_{2} \not \models \varphi_{3}$. Inspired by the approach in [24], it can be shown that any $\mathrm{ADL}^{*}$ formula cannot distinguish between $\mathcal{G}_{1}$ and $\mathcal{G}_{2}$.

In order to show that $\varphi_{1}$ cannot be expressed in LDL-SL[1G], we can adopt the same two CGSs like for $\varphi_{3}$ here. The proof that $\varphi_{4}$ cannot be expressed in BSL[1G] is similar with that for $\varphi_{2}$.

Theorem 5. Inclusion relations among existing strategic logics:

1. $A D L_{s f}^{*} \subsetneq A D L^{*} \subsetneq L D L-S L[1 G] \subsetneq L D L-S L$;

2. $L D L-S L[1 G]_{s f} \subsetneq L D L-S L[1 G]$;

3. $B S L \subsetneq L D L-S L$. 
Proof. By Lemma 1, the star-free logic $\mathrm{ADL}_{s f}^{*}$ (resp. LDL-SL[1G] $]_{s f}$ ) is less expressive than ADL*. (resp. LDL-SL[1G]). One-goal fragment LDL-SL[1G] is obviously less expressive than LDL-SL, due to the restriction about the alternations about strategy variables and agent bindings. Furthermore, the ATL-like fragment ADL* of LDL-SL is less than one-goal fragment LDL-SL[1G] of LDL-SL, since the coalition operators $\langle\langle A\rangle\rangle$ can be specified by the $\wp b$ prefix.

According to Theorems $3-5$, as well as $\mathrm{CL} \subsetneq \mathrm{ATL} \subsetneq \mathrm{ATL}^{*} \subsetneq \mathrm{BSL}[1 \mathrm{G}] \subsetneq \mathrm{SL}$, we can obtain an expressivity graph; see Figure 2.

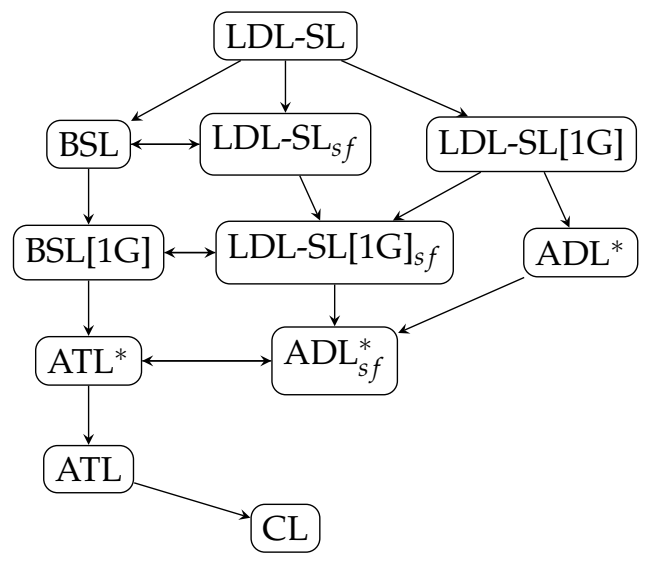

Figure 2. Expressivity Graph.

Here, coalition logic (CL) [29] is a logic, which just has coalition operators without temporal operators.

$$
\varphi::=p|\neg \varphi| \varphi \wedge \varphi \mid\langle\langle A\rangle\rangle .
$$

\section{Positive and Negative Properties for LDL-SL}

In this section, similar with those results about BSL in [30], we state negative/positive results about LDL-SL.

Firstly, as in [30], for LDL-SL, we introduce four basic definitions, including bisimilarity between two CGSs, local isomorphism between two CGSs, state-unwinding, and decisionunwinding.

Definition 12 ([30]). CGSs $\mathcal{G}_{1}=\left\langle\right.$ Act $\left._{1}, W_{1}, \lambda_{1}, \tau_{1}, w_{1}^{0}\right\rangle$ and $\mathcal{G}_{2}=\left\langle A c t_{2}, W_{1}, \lambda_{2}, \tau_{2}, w_{2}^{0}\right\rangle$ are called bisimilar, denoted as $\mathcal{G}_{1} \sim \mathcal{G}_{2}$, if and only if (1) there exists one relation $\sim \subseteq W_{1} \times W_{2}$, named as bisimulation relation, and (2) there exists a function $f: \sim \rightarrow 2^{\text {Act }_{1} \times \text { Act }_{2}}$, named as bisimulation function, satisfying that:

1. $w_{1}^{0} \sim w_{1}^{0}$;

2. for each state pair $\left(w_{1}, w_{2}\right) \in W_{1} \times W_{2}$, if $w_{1} \sim w_{2}$ then

(a) $\lambda_{1}\left(w_{1}\right)=\lambda_{2}\left(w_{2}\right)$;

(b) for each $a c_{1} \in$ Act $t_{1}$, there exists $a c_{2} \in$ Act $_{2}$ satisfying $\left(a c_{1}, a c_{2}\right) \in f\left(w_{1}, w_{2}\right)$;

(c) for each $a c_{2} \in$ Act $t_{2}$, there exists $a c_{1} \in$ Act $t_{1}$ satisfying $\left(a c_{1}, a c_{2}\right) \in f\left(w_{1}, w_{2}\right)$;

(d) for each decision pair $\left(d_{1}, d_{2}\right) \in \hat{f}\left(w_{1}, w_{2}\right)$, it holds that $\tau_{1}\left(w_{1}, d_{1}\right) \sim \tau\left(w_{2}, d_{2}\right)$.

Here, $\hat{f}: \sim \rightarrow 2^{D c_{1} \times D c_{2}}$ is the lifting of function $f$ from actions to decisions, satisfying

$$
\left(d_{1}, d_{2}\right) \in \hat{f}\left(w_{1}, w_{2}\right) \text { iff it holds that }\left(d_{1}(a), d_{2}(a)\right) \in f\left(w_{1}, w_{2}\right), \forall a \in A g .
$$

Obviously, according to the definition of bisimulation relation, the bisimulation of two CGSs can imply the existence of a bismulation between two decisions in them. 
Proposition 1. Suppose that two concurrent game structures $\mathcal{G}_{1}=\left\langle A c t_{1}, W_{1}, \lambda_{1}, \tau_{1}, w_{1}^{0}\right\rangle$ and $\mathcal{G}_{2}=\left\langle\right.$ Act $\left.2, W_{1}, \lambda_{2}, \tau_{2}, w_{2}^{0}\right\rangle$ are bisimilar with a bisimulation relation $\sim$ and a bisimulation relation $f$, for each state pair $\left(w_{1}, w_{2}\right) \in W_{1} \times W_{2}$ with $w_{1} \sim w_{2}$, it holds that:

1. for each $d_{1} \in D c_{1}$, there exists $d_{2} \in D c_{2}$ satisfying that $\left(d_{1}, d_{2}\right) \in \hat{f}\left(w_{1}, w_{2}\right)$;

2. for each $d_{2} \in D c_{2}$, there exists $d_{1} \in D c_{1}$ satisfying that $\left(d_{1}, d_{2}\right) \in \hat{f}\left(w_{1}, w_{2}\right)$.

Next, we define the notion of local isomorphism relation between two CGSs.

Definition 13 ([30]). Two CGSs $\mathcal{G}_{1}=\left\langle A c t_{1}, W_{1}, \lambda_{1}, \tau_{1}, w_{1}^{0}\right\rangle$ and $\mathcal{G}_{2}=\left\langle\right.$ Act $\left._{2}, W_{1}, \lambda_{2}, \tau_{2}, w_{2}^{0}\right\rangle$ are locally isomorphic, denoted as $\mathcal{G}_{1} \cong \mathcal{G}_{2}$, if and only if there exists a bisimulation relation $\sim \subseteq W_{1} \times W_{2}$ between these two CGSs, satisfying that, for each state pair $\left(w_{1}, w_{2}\right) \in W_{1} \times W_{2}$ with $w_{1} \sim w_{2}$

$$
\sim \cap\left(\left\{\tau_{1}\left(w_{1}, d\right): d \in D c_{1}\right\} \times\left\{\tau_{2}\left(w_{2}, d\right): d \in D c_{2}\right\}\right)
$$

is bijective between the successors of $w_{1}$ and those of $w_{2}$.

Now we extend the definition of locally isomorphic to tracks, paths, strategies, and assignments as follows.

Definition 14. Let $\sim($ resp. $f$ ) be a bisimulation relation (resp. function) between two CGSs $\mathcal{G}_{1}=\left\langle A c t_{1}, W_{1}, \lambda_{1}, \tau_{1}, w_{1}^{0}\right\rangle$ and $\mathcal{G}_{2}=\left\langle A c t_{2}, W_{1}, \lambda_{2}, \tau_{2}, w_{2}^{0}\right\rangle$.

- $\quad$ Two tracks $h_{1} \in \operatorname{Trk}\left(\mathcal{G}_{1}\right)$ and $h_{2} \in \operatorname{Trk}\left(\mathcal{G}_{2}\right)$ are locally isomophic, denoted as $h_{1} \sim h_{2}$, if (1) $\operatorname{len}\left(h_{1}\right)=\operatorname{len}\left(h_{2}\right) ;(2) \forall i .0 \leq i<\operatorname{len}\left(h_{1}\right),\left(h_{1}\right)_{i} \sim\left(h_{2}\right)_{i}$ holds.

- Two paths $\pi_{1} \in \operatorname{Path}\left(\mathcal{G}_{1}\right)$ and $\pi_{2} \in \operatorname{Trk}\left(\mathcal{G}_{2}\right)$ are locally isomophic, denoted as $\pi_{1} \sim \pi_{2}$, if $\forall i \in \mathbb{N},\left(\pi_{1}\right)_{i} \sim\left(\pi_{2}\right)_{i}$ holds.

- Two strategies $g_{1} \in \operatorname{Str}\left(\mathcal{G}_{1}\right)$ and $g_{2} \in \operatorname{Str}\left(\mathcal{G}_{2}\right)$ are locally isomophic, denoted as $g_{1} \sim g_{2}$, if $\forall k \in\{1,2\}$ and $h_{k} \in \operatorname{dom}\left(g_{k}\right)$, there exists $h_{3-k} \in \operatorname{dom}\left(g_{3-k}\right)$ with $\pi_{1} \sim \pi_{2}$ satisfying $\left(g_{1}\left(h_{1}\right), g_{2}\left(h_{2}\right)\right) \in f\left(l s t\left(h_{1}\right), l s t\left(h_{2}\right)\right)$.

- Two assignments $\chi_{1} \in \operatorname{Asg}\left(\mathcal{G}_{1}\right)$ and $\chi_{2} \in \operatorname{Asg}\left(\mathcal{G}_{2}\right)$ are locally isomorphic, denoted as $\chi_{1} \sim \chi_{2}$, if (1) $\operatorname{dom}\left(\chi_{1}\right)=\operatorname{dom}\left(\chi_{2}\right)$ and $(2) \chi_{1}(h) \sim \chi_{2}(h), \forall h \in \operatorname{dom}\left(\chi_{2}\right)$.

In Definition 14, obviously, if $\chi_{1} \sim \chi_{2}$ and $g_{1} \sim g_{2}$, then $\chi_{1}\left[x \mapsto g_{1}\right] \sim \chi_{2}\left[x \mapsto g_{2}\right]$. Further, if $\forall i \in\{1,2\}, \chi_{i}$ is a complete $w_{i}$-total assignment, and $w_{1} \sim w_{2}$, then it holds that $\pi_{1} \sim \pi_{2}$ and $\left(\chi_{1}\right)_{\left(\pi_{1}\right)_{\leq k}} \sim\left(\chi_{2}\right)_{\left(\pi_{2}\right)_{\leq k}}, \forall k \in \mathbb{N}$, where $\pi_{i}$ is the $\left(\chi_{i}, w_{i}\right)$-play.

To show whether LDL-SL has tree model properties, consider two unwinding forms of concurrent game structures; one is about state-unwinding, and another is about decisionunwinding.

Definition 15 ([30]). Given a $C G S \mathcal{G}=\left\langle A c t, W, \lambda, \tau, w^{0}\right\rangle$, the state-unwinding of $\mathcal{G}$ is the new CGS $\mathcal{G}_{s u}=\left\langle A c, W_{s u}, \lambda_{s u}, \tau_{s u}, \epsilon\right\rangle$, where

- $W_{s u}=\left\{h_{\geq 1} \in W^{*}: h \in \operatorname{Trk}\left(\mathcal{G}, w^{0}\right)\right\} ;$

- $\tau_{s u}(h, d)=h \cdot \tau\left(\operatorname{last}\left(w^{0} \cdot h\right), d\right)$, here $d \in D c$;

- there exists a surjective function surj : $W_{s u} \rightarrow W$, satisfying that for each $h \in W_{s u}$ and $d \in D c,(1) \operatorname{surj}(h)=\operatorname{last}\left(w^{0} \cdot h\right) ;(2) \lambda_{s u}(h)=\lambda(\operatorname{surj}(h))$.

From Definition 15, the state-unwinding $\mathcal{G}_{s u}$ of a CGS $\mathcal{G}=\left\langle A c t, W, \lambda, \tau, w^{0}\right\rangle$ is a tree, whose direction set is just the set $W$ of states in $\mathcal{G}$.

Definition 16 ([30]). Given a $C G S \mathcal{G}=\left\langle A c t, W, \lambda, \tau, w^{0}\right\rangle$, the decision-unwinding of $\mathcal{G}$ is the new $\operatorname{CGS} \mathcal{G}_{d u}=\left\langle A c t, W_{d u}, \lambda_{d u}, \tau_{d u}, \epsilon\right\rangle$, where

- $\quad W_{d u}=D c^{*}$ and $\tau_{d u}(h, d)=h \cdot d$;

- there exists a surjective function surj $: W_{d u} \rightarrow W$, satisfying that for each $h \in W_{d u}$ and $d \in D c,(1) \operatorname{surj}(\epsilon)=w^{0} ;(2) \operatorname{surj}\left(\tau_{d u}((h, d))=\tau(\operatorname{surj}(h), d) ;(3) \lambda_{d u}(h)=\lambda(\operatorname{surj}(h))\right.$. 
From Definition 16, the decision-unwinding $\mathcal{G}_{d u}$ of a CGS $\mathcal{G}=\left\langle A c t, W, \lambda, \tau, w^{0}\right\rangle$ is a tree, whose direction set is just the set $D c$ (i.e., $A c t^{A g}$ ) in $\mathcal{G}$.

Theorem 6 ([30]). Given a CGS $\mathcal{G}$, the following properties hold:

1. $\mathcal{G}$ and its state-unwinding $\mathcal{G}_{\text {su }}$ are locally isomorphic;

2. $\mathcal{G}$ and decision-unwinding $\mathcal{G}_{d u}$ are bisimilar;

3. there exists a $C G S \mathcal{G}^{\prime}$, satisfying that $\mathcal{G}^{\prime}$ and $\mathcal{G}_{d u}^{\prime}$ are not locally isomorphic.

We note that any CGS $\mathcal{G}$ just has a unique associated state-unwinding $\mathcal{G}_{\text {su }}$ and a unique associated decision-unwinding $\mathcal{G}_{d u}$.

For BSL logic, the following negative properties hold.

Theorem 7 ([30]). Four negative properties for BSL:

- it holds that BSL is not decision-unwinding invariant;

- $\quad$ it holds that BSL does not have the bounded tree model property;

- it holds that BSL does not have the finite model property;

- $\quad$ it holds that BSL is not bisimulation invariant.

These negative results can be extended into LDL-SL.

Theorem 8. Four negative properties for LDL-SL:

- it holds that LDL-SL is not decision-unwinding invariant;

- it holds that LDL-SL does not have the bounded tree model property;

- $\quad$ it holds that LDL-SL does not have the finite model property;

- $\quad$ it holds that LDL-SL is not bisimulation invariant.

Proof. By Theorems 2 and 7, these results are the same as those for BSL.

Similar with those positive properties for BSL [30], the following properties also hold for LDL-SL.

Theorem 9. Three positive properties for LDL-SL:

1. it holds that LDL-SL is local isomorphism invariant;

2. it holds that LDL-SL is state-unwinding invariant;

3. it holds that LDL-SL has the unbounded tree model property.

Proof. For item 1:

For any LDL-SL formula $\varphi$, given any two CGSs $\mathcal{G}_{1}$ and $\mathcal{G}_{2}$ with $\mathcal{G}_{1} \cong \mathcal{G}_{2}$, two states $w_{1} \in W_{1}$ and $w_{2} \in W_{2}$ with $w_{1} \sim w_{2}$, two assignments $\chi_{1} \in \operatorname{Asg}\left(\mathcal{G}_{1}, w_{1}\right)$, and $\chi_{2} \in \operatorname{Asg}\left(\mathcal{G}, w_{2}\right)$ with $\chi_{1} \sim \chi_{2}$, here $\operatorname{free}(\varphi) \subseteq \operatorname{dom}\left(\chi_{1}\right)=\operatorname{dom}\left(\chi_{2}\right)$, we inductively show that

$$
\mathcal{G}_{1}, \chi_{1}, w_{1} \models \varphi \quad \text { if and only if } \quad \mathcal{G}_{2}, \chi_{2}, w_{2}=\varphi .
$$

From the bisimulation definition and the inductive hypothesis, the cases of atoms and Boolean connectives are easy. As for the cases of existential quantification $\langle\langle x\rangle\rangle$ and agent binding $(a, x)$, the proofs are the same as those in [30]. Here we just show the case of $\mathbf{E} \psi$, here $\psi$ is a path formula. $\mathcal{G}_{1}, \chi_{1}, w_{1} \models \mathbf{E} \psi$ iff there exists a $\pi \in \operatorname{out}\left(\mathcal{G}_{1}, \chi_{1}, w_{1}\right)$ such that $\mathcal{G}_{1}, \chi_{1}, \pi_{1}, 0 \models \psi$.

That means we should mutually show with state formulas by induction, i.e.,

$$
\mathcal{G}_{1}, \chi_{1}, \pi_{1}, i=\psi \quad \text { if and only if } \mathcal{G}_{2}, \chi_{2}, \pi_{2}, i \models \psi .
$$

For the case $\psi=\varphi^{\prime}: \mathcal{G}_{1}, \chi_{1}, \pi_{1}, i \models \varphi^{\prime}$ iff $\mathcal{G}_{1}, \chi_{1},\left(\pi_{1}\right)_{i} \models \varphi^{\prime}$ iff $\mathcal{G}_{2}, \chi_{2},\left(\pi_{2}\right)_{i} \models \varphi_{2}$ by induction iff $\mathcal{G}_{2}, \chi_{2}, \pi_{2}, i=\varphi^{\prime}$. 
For the cases of Boolean connectives, these are easy from the definitions and the inductive hypothesis.

For the case $\psi=\langle\rho\rangle \psi^{\prime}$ : we need to show the following by induction,

$$
(i, j) \in \mathcal{R}\left(\mathcal{G}_{1}, \rho, \pi_{1}, \chi_{1}\right) \quad \text { if and only if }(i, j) \in \mathcal{R}\left(\mathcal{G}_{2}, \rho, \pi_{2}, \chi_{2}\right) \text {. }
$$

For case $\rho=\Phi:(i, j) \in \mathcal{R}\left(\mathcal{G}_{1}, \Phi, \pi_{1}, \chi_{1}\right)$ iff $j=i+1$ and $\mathcal{G}_{1}, \chi_{1}, \pi_{1}, i=\Phi$ by definition iff $j=i+1$ and $\mathcal{G}_{2}, \chi_{2}, \pi_{2}, i=\Phi$.

For case $\rho=\psi$ ?: $(i, j) \in \mathcal{R}\left(\mathcal{G}_{1}, \psi ?, \pi_{1}, \chi_{1}\right)$ iff $j=i$ and $\mathcal{G}_{1}, \chi_{1}, \pi_{1}, i \models \psi$ by definition iff $j=i$ and $\mathcal{G}_{2}, \chi_{2}, \pi_{2}, i \models \psi$ by induction.

For case $\rho=\rho_{1}+\rho_{2}:(i, j) \in \mathcal{R}\left(\mathcal{G}_{1}, \rho_{1}+\rho_{2}, \pi_{1}, \chi_{1}\right)$ iff $(i, j) \in \mathcal{R}\left(\mathcal{G}_{1}, \rho_{1}, \pi_{1}, \chi_{1}\right)$ or $(i, j) \in \mathcal{R}\left(\mathcal{G}_{1}, \rho_{2}, \pi_{1}, \chi_{1}\right)$ iff $(i, j) \in \mathcal{R}\left(\mathcal{G}_{2}, \rho_{1}, \pi_{2}, \chi_{2}\right)$ or $(i, j) \in \mathcal{R}\left(\mathcal{G}_{2}, \rho_{2}, \pi_{2}, \chi_{2}\right)$ by induction iff $(i, j) \in \mathcal{R}\left(\mathcal{G}_{2}, \rho_{1}+\rho_{2}, \pi_{2}, \chi_{2}\right)$.

For case $\rho=\rho_{1} ; \rho_{2}:(i, j) \in \mathcal{R}\left(\mathcal{G}_{1}, \rho_{1} ; \rho_{2}, \pi_{1}, \chi_{1}\right)$ iff there exists $k, i \leq k \leq j$, satisfying that $(i, k) \in \mathcal{R}\left(\mathcal{G}_{1}, \rho_{1}, \pi_{1}, \chi_{1}\right)$ and $(k, j) \in \mathcal{R}\left(\mathcal{G}_{1}, \rho_{2}, \pi_{1}, \chi_{1}\right)$ iff there exists $\mathrm{k}, i \leq k \leq j$, satisfying that $(i, k) \in \mathcal{R}\left(\mathcal{G}_{2}, \rho_{1}, \pi_{2}, \chi_{2}\right)$ and $(k, j) \in \mathcal{R}\left(\mathcal{G}_{2}, \rho_{2}, \pi_{2}, \chi_{2}\right)$ by induction iff $(i, j) \in \mathcal{R}\left(\mathcal{G}_{2}, \rho_{1} ; \rho_{2}, \pi_{2}, \chi_{2}\right)$.

For case $\rho=\rho_{1}^{*}:(i, j) \in \mathcal{R}\left(\mathcal{G}_{1}, \rho_{1}^{*}, \pi_{1}, \chi_{1}\right)$ iff $j=i$ or $(i, j) \in \mathcal{R}\left(\mathcal{G}_{1}, \rho_{1} ; \rho_{1}^{*}, \pi_{1}, \chi_{1}\right)$ iff $j=i$ or $(i, j) \in \mathcal{R}\left(\mathcal{G}_{2}, \rho_{2} ; \rho_{2}^{*}, \pi_{1}, \chi_{1}\right)$ by induction iff $(i, j) \in \mathcal{R}\left(\mathcal{G}_{2}, \rho_{1}^{*}, \pi_{2}, \chi_{2}\right)$.

Therefore, it implies that LDL-SL is indeed invariant under local isomorphism.

For item 2: by item 1 in Theorem 6, for any CGS $\mathcal{G}$, it holds that $\mathcal{G} \cong \mathcal{G}_{\text {su }}$. So by item 1 , each LDL-SL sentence $\varphi$ is an invariant for CGS $\mathcal{G}$ and its state-unwinding $\mathcal{G}_{s u}$.

For item 3: let the LDL-SL sentence $\varphi$ be satisfiable. Therefore, there exists one CGS $\mathcal{G} \models \varphi$, and by item 2 , it holds that $\mathcal{G}_{s u} \models \varphi$. Since $\mathcal{G}_{s u}$ is a tree model, this means that LDL-SL has the (unbounded) tree model property.

\section{Complexities of Model Checking}

In this section, we analyse the computational complexities of the model checking problems for these strategic logics. Firstly, we present the definition about the model checking problem. Secondly, we study the model-checking complexities for ADL* , LDLSL[1G], and LDL-SL. Then we apply expressivity results to infer other logics' modelchecking complexities. Due to space restriction, we omit the introduction about automaton theory; please refer to, e.g., [31].

Let $|\mathcal{G}|$ (resp. $|\varphi|$ ) denote the number of transitions in $\mathcal{G}$ (resp. the length of $\varphi$ ).

Problem 1 ( Model-Checking Problem (MCP) for Strategic Logic)). given a concurrent game structure CGS $\mathcal{G}$, a sentence $\varphi$ in strategic logic $\mathcal{L}$, and a state $w$, decide whether $\mathcal{G}, w=\varphi$.

\subsection{Model-Checking for $A D L^{*}$}

Before considering the $\mathrm{MCP}$ for $\mathrm{ADL}^{*}$, remember that a state formula in a test in a $\mathrm{ADL}^{*}$ state formula $\varphi$ is also a state subformula of $\varphi$.

Theorem 10. The computational complexity of model-checking for ADL* is 2EXPTIME-complete, in time polynomial w.r.t. the size of CGS model and double exponential in the size of ADL* formula.

Proof. Firstly, because the MCP for ATL* is 2EXPTIME-complete [5], which can be linearly encoded by that for ADL* by Lemma 5, then the MCP for ADL* is 2EXPTIME-hard. Next we show that the complexity of model checking for $\mathrm{ADL}^{*}$ is in 2EXPTIME.

Given a CGS model $\mathcal{G}=\left\langle A c, W, \lambda, \tau, w^{0}\right\rangle$ and an $\mathrm{ADL}^{*}$ formula $\varphi$, as in the modelchecking algorithm for CTL, we adopt the labelling algorithm, in a bottom-up fashion, starting from the innermost state subformulas of $\varphi$. We label each state $w$ of $\mathcal{G}$ by all state subformulas of $\varphi$ that are satisfied in $w$. To give this algorithm, we only consider the case $\varphi=\langle\langle A\rangle\rangle \psi$, for each subformula $\varphi^{\prime}$ such as $\langle\langle B\rangle\rangle \psi^{\prime}$ in $\psi$, introduce a new atomic proposition $p_{\varphi^{\prime}}$ in $\mathcal{G}$, where for each state $w, p_{\varphi^{\prime}} \in \lambda(w)$ iff $\mathcal{G}, w \models \varphi^{\prime}$. Therefore, assume that $\psi$ is just an LDL formula. 
Now we mainly consider $\varphi=\langle\langle A\rangle\rangle \psi$, where $\psi$ is an LDL formula.

1. Construct a Büchi tree automaton $\mathcal{A}_{\mathcal{G}, w, A}$ as in [5].

Here, $\mathcal{A}_{\mathcal{G}, w, A}$ accepts exactly the $(w, A)$-execution trees, which are trees induced by $\operatorname{out}\left(w, g_{A}\right)$, where $g_{A}$ is a collective strategy of $A$. Automaton $\mathcal{A}_{\mathcal{G}, w, A}$ is bounded by $O(|\mathcal{G}|)$.

2. Construct a Rabin tree automaton $\mathcal{A}_{\mathrm{A} \psi}$.

Here, $\mathcal{A}_{\mathrm{A} \psi}$ accepts all trees that satisfy the $\mathrm{CDL}^{*}$ formula $\mathrm{A} \psi \cdot \mathcal{A}_{\psi}$ has $2^{2^{\mathrm{O}(|\psi|)}}$ states and $2^{O(|\psi|)}$ Rabin pairs.

- $\quad$ For LDL formula $\psi$, construct an alternating Büchi automaton (ABA) $\mathcal{A}_{\psi}$ with linearly many states in $\psi$ [21].

- Turn automaton $\mathcal{A}_{\psi}$ into a nondeterministic Büchi automaton (NBA) $\mathcal{A}_{\psi}^{\prime}$ of exponential size of $|\psi|$ [32].

- Turn automaton $\mathcal{A}_{\psi}^{\prime}$ into a deterministic Rabin automaton $\mathcal{A}_{\psi}^{\prime \prime}$ (DRA) of doubleexponential size of $|\psi|$ [33].

- $\quad$ According to automaton $\mathcal{A}_{\psi}^{\prime \prime}$, build the Rabin tree automaton $\mathcal{A}_{\mathbf{A} \psi}$ for $\mathbf{A} \psi$ in a relatively obvious method; this tree automaton is designed to simply run the deterministic string automaton for $\psi$ down every path from the root.

3. Construct product automaton $\mathcal{A}=\mathcal{A}_{\mathrm{A} \psi} \times \mathcal{A}_{\mathcal{G}, w, A}$, which is a Rabin tree automaton with $n=O\left(\left|\mathcal{A}_{\mathrm{A} \psi}\right| \cdot\left|\mathcal{A}_{\mathcal{G}, w, A}\right|\right)$ many states and $r=2^{O(|\psi|)}$ many Rabin pairs.

The decidable problem is to determine whether $L(\mathcal{A}) \neq \varnothing$ can be done in time $O(n \cdot r)^{3 r}[34,35]$.

The automata $\mathcal{A}$ is a Rabin tree automaton that accepts precisely the $(w, A)$-execution trees that satisfy $\mathbf{A} \psi$.

Since $\mathcal{G}, w \models\langle\langle A\rangle\rangle \psi$ iff there is a collective strategy $g_{A}$ so that all $w$-computations in $\operatorname{out}\left(w, g_{A}\right)$ satisfy $\psi$. Since each $\langle w, A\rangle$-execution tree corresponds to a set $g_{A}$ of strategies, it follows that $\mathcal{G}, w \models\langle\langle A\rangle\rangle \psi$ iff the product automaton is nonempty. According to the above steps, the whole algorithm runs in polynomial time in the size of model and double exponential time in the size of formula.

In fact, according to the above algorithm, since both CTL* satisfiability-checking [36] and module-checking [37] problems are 2EXPTIME-complete, then CDL* satisfiability and module checking problems are also 2EXPTIME-complete.

\subsection{Model-Checking for LDL-SL[1G]}

To give a model-checking algorithm for LDL-SL[1G], we adopt a similar approach proposed in [4], which is used to show that $S L_{1 G}[\mathcal{F}]$ model checking is 2EXPTIME-complete.

First, we introduce the concept of concurrent multi-player parity game (CMPG) $\mathcal{P}=$ $\left(A g, A c, S, s_{0}, \mathrm{p}, \Delta\right)[38]$, here $A g=\{1, \cdots, n\}, \Delta$ is a transition function, and $\mathrm{p}: S \rightarrow N$ is a priority function. In a CMPG $\mathcal{P}$, there are $n$ agents playing concurrently with infinite rounds. Informally, in a CMPG, if there exists one strategy for agent 0, s.t., for any strategy for agent 1 , there exists one strategy for agent 2 , and so forth, which make all the induced plays satisfy the parity condition, and then the existential coalition wins; otherwise, the universal coalition wins.

In a $C M P G, \mathcal{P}=\left(A g, A c, S, s_{0}, p, \Delta\right)$, the winners of which can be determined in polynomial-time with respect to $|S|$ and $|A c|$ and exponential-time with respect to $|A g|$ and $\max \mathrm{p}[38]$.

Theorem 11. The MCP for LDL-SL[1G] is 2EXPTIME-complete.

Proof. Firstly, Hardness follows from the fact that the MCP for BSL[1G] is 2EXPTIMEcomplete [24]. Then, we consider the lower bound of LDL-SL[1G].

Consider a CGS $\mathcal{G}=\left\langle A c, W, \lambda, \tau, w_{0}\right\rangle$ and a LDL-SL[1G] sentence $\varphi$. As in $\mathrm{ADL}^{*}$, we present a labelling algorithm to solve LDL-SL[1G] model checking. Like in [4], here we just 
consider the case sentence $\wp b \psi$, where quantifiers perfectly alternate between existential and universal $\left\langle\left\langle x_{1}\right\rangle\right\rangle \llbracket x_{2} \rrbracket \cdots \llbracket x_{n} \rrbracket$, and $\psi$ is an LDL formula. Now $\psi$ can be interpreted over paths of the pointed Kripke model $M=\left(W, R, \lambda, w_{0}\right)$, where $R=\left\{\left(w_{1}, w_{2}\right) \mid \exists d \in\right.$ $\left.A c^{A g}, w_{2}=\tau\left(w_{1}, d\right)\right\}$.

In [21], for LDL formula $\psi$, construct an $\mathrm{ABA} \mathcal{B}_{\psi}$ with linearly many states in $\psi$, and then turn $\mathcal{B}_{\psi}$ into an NBA $\mathcal{A}_{\psi}$ of exponential size of $|\psi|$ [32]. Combining $\mathcal{A}_{\psi}$ with Kripke model $M$, we get a new NBA $\mathcal{A}_{M, \psi}$, which accepts exactly all the infinite paths $\pi$ of $M$ s.t. $\pi, 0 \models \psi$. Then, by [39], we convert $\mathcal{A}_{M, \psi}$ into a deterministic parity automaton (DPA) $\mathcal{A}_{M, \psi}=\left(W, Q, q_{0}, \delta, \mathrm{p}\right)$ of size in $2^{2^{O(|\psi|)}}$ and index bounded by $2^{\mathrm{O}(|\psi|)}$.

Now as in [4], combining CGS $\mathcal{G}$ with $\mathcal{A}_{M, \psi}$, we use the same approach to define the following CMPG $\mathcal{P}=\left(A g^{\prime}, A c, S, s_{0}, \mathrm{p}, \Delta^{\prime}\right)$, where $A g^{\prime}$ is a set of agents, one for every variable occurring in $\wp ; S=W \times Q$. Firstly, game $\mathcal{P}$ emulates a path $\pi$ generated in $\mathcal{G}$; secondly, if the generated path $\pi$ in $\mathcal{G}$ is read, then the game emulates the execution of $\mathcal{A}_{M, \psi}$. Hence, each execution $(\pi, l) \in W^{\omega} \times Q^{\omega}$ in game $\mathcal{P}$ satisfies the parity condition determined by the $\mathrm{p}^{\prime}$ in $\mathcal{G}$ iff $\pi, 0=\psi$. In addition, because $\mathcal{A}_{M, \psi}$ is deterministic, for each possible track $h \in \operatorname{Tr}(\mathcal{G})$, there is one unique partial path $l_{h}$ that makes the partial execution $\left(h, l_{h}\right)$ possible in $\mathcal{P}$. This makes the strategies from $w_{0}$ in $\operatorname{Str}(\mathcal{G})$ one-to-one with the strategies from $s_{0}$ in $\operatorname{Str}(\mathcal{P})$. Then $\mathcal{P}$ has a winning strategy if and only if $\mathcal{G}, w_{0} \models \wp b \psi$.

As for complexity, the size of $\mathcal{P}$ is $O(|W| \cdot|Q|)$, where $W$ is the state space of $\mathcal{G}$ and $|Q|=2^{2^{O(|\psi|)}}$, i.e., doubly exponential in the size of $\psi$. Since $\mathcal{A}_{M, \psi}$ results from one NGBW $\mathcal{B}_{M, \psi}$, whose size is $2^{O}(|\psi|)$, transformed into a DPW, which needs another exponential in $\psi$. Moreover, since the transformation from an NGBW to a DPW just needs $2^{O(|\psi|)}$ priorities, so the number of priorities in $\mathcal{P}$ is $2^{O(|\psi|)}$. Hence, the constructed CMPG $\mathcal{P}$ can be solved in time polynomial with respect to the size of the CGS model $\mathcal{G}$ and double exponential in formula $|\psi|$.

In fact, according to Theorem 11, since ADL* and BSL[1G] can both be linearly embedded into LDL-SL[1G], then the MCPs for both logics are in 2EXPTIME.

\subsection{Model-Checking for LDL-SL}

Since the MCP for BSL is non-elementary-complete [24], in addition to Theorem 2, then the lower bound of the MCP for LDL-SL is non-elementary.

Theorem 12. The MCP for LDL-SL is non-elementary-hard.

As for the upper bound of MCP for LDL-SL, we conjecture that we could reduce the MCP for LDL-SL into that for QCTL* under the tree semantics [40], inspired by the approach proposed in [41].

Conjecture 13. The MCP for LDL-SL is non-elementary-complete.

In addition, since ATL*, BSL, and BSL[1G] can be linearly embedded into their corresponding star-free strategic logics $\mathrm{ADL}_{s f}^{*}, \mathrm{LDL}_{-S L_{s f}}$, and LDL-SL[1G] $]_{s f}$, respectively, MCPs for ATL* [5] and BSL[1G] [24] are 2EXPTIME-complete, and MCP for BSL is nonelementary-complete [24], then the following holds.

Corollary 3. The MCPs for both $A D L_{s f}^{*}$ and $L D L-S L[1 G]_{s f}$ are $2 E X P T I M E-h a r d$. The MCP for $L D L-S L_{s f}$ is non-elementary-hard.

Although similar expressive power by Theorem 3, we do not know how to linearly translate star-free logics to the corresponding logics. For the time being, the upper bounds of these star-free strategic logics are not known.

The main complexity results about the MCPs are given in Table 1. 
Table 1. Complexity of Model Checking for Strategic Logics.

\begin{tabular}{|c|c|}
\hline Strategic Logics & Complexity of Model-Checking \\
\hline $\mathrm{CL}$ & PTIME-complete [42] \\
\hline ATL & PTIME-complete [5] \\
\hline ATL* & 2EXPTIME-complete [5] \\
\hline $\mathrm{ADL}_{s f}^{*}$ & 2EXPTIME-hard (Corollary 3) \\
\hline $\mathrm{ADL}^{*}$ & 2EXPTIME-complete (Theorem 10) \\
\hline BSL[1G] & 2EXPTIME-complete ([24]) \\
\hline BSL & non-elementary-complete ([24]) \\
\hline LDL-SL $_{s f}[1 \mathrm{G}]$ & 2EXPTIME-hard (Corollar 3) \\
\hline${\mathrm{LDL}-S L_{s} f}$ & non-elementary-hard (Corollary 3) \\
\hline LDL-SL[1G] & 2EXPTIME-complete (Theorem 11) \\
\hline LDL-SL & non-elementary-hard (Theorem 12) \\
\hline
\end{tabular}

\section{Conclusions and Future Work}

In this paper, we propose logic LDL-SL, an expressive new strategic logic based on linear dynamic logic, which can naturally express $\omega$-regular properties. This logic is a branching-time extension of SL based on linear-time temporal logic. We show that LDL-SL is more expressive than SL, whose model-checking complexity is non-elementarycomplete. Moreover, based on LDL, we define similar fragments of LDL-SL, which are more expressive than corresponding strategic logics based on LTL. However, all these fragments have the same model checking complexity, i.e., are 2EXPTIME-complete. At the same time, we define star-free-like strategic logics, based on star-free regular expressions. We show that these logics have the same expressivity as those corresponding strategic logics based on LTL or CTL*.

In short, based on LDL, we propose a new class of strategic logics. These logics have the same model-checking complexities as, but more expressivity than, current mainstream strategic logics. Furthermore, these logics can extend the application areas in multi-agent systems.

However, until now, the upper bounds of LDL-SL and its star-free fragments (ADL $\mathrm{Af}^{*}$, LDL-SL[1G $]_{s f}$, and LDL-SL ${ }_{s f}$ ) are not known. In future, we will study the compact bounds of these logics. As in [43,44], we will consider concrete implementations about these model checking problems. In addition, here we just consider perfect recall strategies in multi-agent concurrent games with complete information. Next, we will further study these new proposed strategic logics under incomplete information [45-47], where the strategies of agents maybe memoryless or perfect recall [48]. In this paper, we present formal frameworks and show technical results; in the future, we will also present case studies or practical applications to illustrate these theories, such as information security [49], solving winning strategies [50], and voting protocol [51].

Author Contributions: Conceptualization, L.X. and S.G.; methodology, L.X. and S.G; validation, L.X. and S.G.; formal analysis, L.X.; investigation, L.X.; resources, L.X.; writing—original draft preparation, L.X.; writing-review and editing, L.X. and S.G.; project administration, S.G. All authors have read and agreed to the published version of the manuscript.

Funding: This research received no external funding.

Institutional Review Board Statement: Not applicable.

Informed Consent Statement: Not applicable.

Data Availability Statement: Not applicable.

Acknowledgments: Our deepest gratitude goes to the anonymous reviewers for their careful work and thoughtful suggestions that have helped improve this paper substantially. 
Conflicts of Interest: The authors declare no conflict of interest.

$\begin{array}{ll}\begin{array}{l}\text { Abbreviations } \\ \text { The following abbreviations are used in this manuscript }\end{array} \\ \text { ADL* } & \text { Alternating-time dynamic strategic logic } \\ \text { AMC } & \text { Alternating-time mu-calculus } \\ \text { ATL/ATL* } & \text { (Flat) alternating-time temporal logic } \\ \text { BSL } & \text { Branching version of Strategy Logic } \\ \text { CDL* } & \text { Computational-tree dynamic logic } \\ \text { CGS } & \text { Concurrent game structure } \\ \text { CL } & \text { Coalition logic } \\ \text { CMPG } & \text { Concurrent multi-player parity game } \\ \text { CTL/CTL* } & \text { (Flat) computational tree logic } \\ \text { LDL } & \text { Linear dynamic logic } \\ \text { LDL-SL } & \text { LDL-based Strategy Logic } \\ \text { LTL } & \text { Linear-time temporal logic } \\ \text { MCP } & \text { Model checking problem } \\ \text { PDL } & \text { Propositional Dynamic Logic } \\ \text { QCTL* } & \text { Quantified computational tree logic } \\ \text { sf } & \text { Star-free } \\ \text { SL } & \text { Strategy Logic }\end{array}$

\section{References}

1. Pnueli, A. The temporal semantics of concurrent programs. Theor. Comput. Sci. 1981, 13, 45-60.

2. Clarke, E.M.; Emerson, A. Design and Synthesis of Synchronization Skeletons Using Branching-time Temporal Logic. In Logic of Programs: Workshop on Logic of Programs; Kozen, D.C., Ed.; Springer: Berlin, Heidelberg, 1982; Volume 131, pp. 45-60.

3. Emerson, E.A.; Halpern, J.Y. "Sometimes" and "Not Never" revisited: On branching versus linear time temporal logic. J. ACM 1986, 33, 1, 151-178.

4. Bouyer, P.; Kupferman, O.; Markey, N.; Maubert, B.; Murano, A.; Perelli, G. Reasoning about Quality and Fuzziness of Strategic Behaviours. In Proceedings of the Twenty-Eighth International Joint Conference on Artificial Intelligence Main Track, Macao, China, 10-16 August 2019; pp. 1588-1594.

5. Alur, R.; Henzinger, T.A. Alternating-time temporal logic. J. ACM 2002, 49, 5, 672-713.

6. Chatterjee, K.; Henzinger, T.A.; Piterman, N. Strategy logic. Inf. Comput. 2010, 208, 6, 677-693.

7. Mogavero, F.; Murano, A.; Vardi, M.Y. Reasoning About Strategies. In Proceedings of the IARCS Annual Conference on Foundations of Software Technology and Theoretical Computer Science, (FSTTCS-2010), Chennai, India, 15-18 December 2010; pp. 133-144.

8. Aminof, B.; Malvone, V.; Murano, A.; Rubin, S. Graded Strategy Logic: Reasoning about Uniqueness of Nash Equilibria. In Proceedings of the AAMAS 2016, Singapore, 9-13 May 2016; pp. 133-144.

9. Bozzelli, L.; Murano, A.; Sorrentino, L. Alternating-time temporal logics with linear past. Theor. Comput. Sci. 2020, 813, 199-217.

10. Belardinelli, F.; Knight, S.; Lomuscio, A.; Maubert, B.; Murano, A.; Rubin, S. Reasoning About Agents That May Know Other Agents' Strategies. In Proceedings of the IJCAI 2021, Montreal, QC, Canada, 19-27 August 2021; pp. 1787-1793.

11. Pnueli, A. The Temporal Logic of Programs. In Proceedings of 18th Annual Symposium on Foundations of Computer Science , Providence, RI, USA, 31 October-1 November 1977; pp. 46-57.

12. Emerson, E.A.; Halpern, J.Y. Decision Procedures and Expressiveness in the Temporal Logic of Branching Time. In Proceedings of the 14th Annual ACM Symposium on Theory of Computing, San Francisco, CA, USA, 5-7 May 1982; pp. 169-180.

13. Kozen, D. Results on the propositional mu-calculus. Theor. Comput. Sci. 1983, 27, 333-354.

14. Wolper, P. Temporal logic can be more expressive. Inf. Control 1983, 56, 72-99.

15. Armoni, R; Fix, L.; Flaisher, A.; Gerth, R.; Ginsburg, B.; Kanza, T.; Landver, A.; Mador-Haim, S.; Singerman, E.; Tiemeyer, A.; et al. The ForSpec Temporal Logic: A New Temporal Property-Specification Language. In Tools and Algorithms for the Construction and Analysis of Systems, Proceedings of the 8th International Conference, TACAS 2002, Held as Part of the Joint European Conference on Theory and Practice of Software, ETAPS 2002, Grenoble, France, 8-12 April 2002; Springer: Berlin, Heidelberg, 2002; pp. $296-211$.

16. Henriksen, J.G.; Thiagarajan, P.S. Dynamic linear time temporal logic. Ann. Pure Appl. Logic 1999, 96, $187-207$.

17. Vardi, M.Y. The Rise and Fall of Linear Time Logic. In Proceedings of the Second International Symposium on Games, Automata, Logics and Formal Verification, GandALF 2011, Minori, Italy, 15-17 June 2011.

18. Fischer, M.J.; Ladner, R.E. Propositional dynamic logic of regular programs. J. Comput. Syst. Sci. 1979, 18, $194-211$.

19. Büchi, J.R.; Landweber, L.H. Definability in the monadic second-order theory of successor. J. Symb. Log. 1969, 34, 166-170.

20. De Giacomo, G.; Vardi, M.Y. Linear Temporal Logic and Linear Dynamic Logic on Finite Traces. In Proceedings of the IJCAI, Beijing, China, 3-9 August 2013; pp. 854-860. 
21. Faymonville, P.; Zimmermann, M. Parametric linear dynamic logic. Inf. Comput. 2017, 253, 237-256.

22. Liu, Z.; Xiong, L.; Liu, Y.; Lespérance, Y.; Xu, R.; Shi, H. A Modal Logic for Joint Abilities under Strategy Commitments. In Proceedings of IJCAI, Yokohama, Japan, 7-15 January 2020; pp. 1805-1812.

23. Belardinelli, F.; Lomuscio, A.; Murano, A.; Rubin, S. Decidable Verification of Multi-agent Systems with Bounded Private Actions. In Proceedings of the 17th International Conference on Autonomous Agents and MultiAgent Systems, AAMAS, Stockholm, Sweden, 10-15 July 2018; pp. 1865-1867.

24. Mogavero, F.; Murano, A.; Perelli, G.; Vardi, M.Y. Reasoning about strategies: On the model-checking problem. ACM Trans. Comput. Log. 2014, 34, 1-47.

25. Kong, J. MCMAS-Dynamic: Symbolic Model Checking for Linear Dynamic Logic and Several Temporal and Epistemic Extensions. Ph.D. Thesis, Imperial College London, London, UK, 2016.

26. Knight, S.; Maubert, B. Dealing with Imperfect Information in Strategy Logic. Available online: https://arxiv.org/abs/1908.02488 (accessed on 7 August 2019).

27. Thomas, W. Star-free regular sets of $\omega$-sequences. Inf. Control 1979, 42, 148-156.

28. Gabbay, D.M.; Pnueli, A.; Shelaho, S.; Shelah, J. On the Temporal Basis of Fairness. In Proceedings of the Conference Record of the Seventh Annual ACM Symposium on Principles of Programming Languages, Las Vegas, NV, USA, 28-30 January 1980; pp. 163-173.

29. Pauly, M. A modal logic for coalitional power in games. J. Log. Comput. 2002, 12, 149-166.

30. Mogavero, F. Logics in Computer Science-A Study on Extensions of Temporal and Strategic Logics; Atlantis Studies in Computing 3; Atlantis Press: Paris, France, 2013; pp. 85-101.

31. Thomas, W. Automata on infinite objects. In Handbook of Theoretical Computer Science, Volume B: Formal Models and Semantics; MIT Press: Cambridge, MA, USA, 1990; pp. 133-191.

32. Miyano, S.; Hayashi, T. Alternating finite automata on omega-words. Theor. Comput. Sci. 1984, 32, 321-330.

33. Schewe, S. Tighter Bounds for the Determinisation of Büchi Automata. In Proceedings of the Foundations of Software Science and Computational Structures, 12th International Conference, York, UK, 22-29 March 2009; pp. 167-181.

34. Emerson, E.A.; Jutla, C.S. The Complexity of Tree Automata and Logics of Programs (Extended Abstract). In Proceedings of the 29th Annual Symposium on Foundations of Computer Science, White Plains, New York, NY, USA, 24-26 October 1988; pp. 328-337.

35. Pnueli, A.; Rosner, R. On the Synthesis of a Reactive Module. In Proceedings of the Conference Record of the Sixteenth Annual ACM Symposium on Principles of Programming Languages, Austin, TX, USA, 11-13 January 1989; pp. 179-190.

36. Emerson, E.A. Temporal and modal logic. In Handbook of Theoretical Computer Science, Volume B: Formal Models and Semantics; MIT Press: Cambridge, MA, USA, 1990; pp. 995-1072.

37. Kupferman, O.; Vardi, M.Y.; Wolper, W. Module checking. Inf. Comput. 2001, 164, 2, 322-344.

38. Malvone, V.; Murano, A.; Sorrentino, L. Concurrent Multi-Player Parity Games. In Proceedings of the 2016 International Conference on Autonomous Agents \& Multiagent Systems, Singapore, 9-13 May 2016; pp. 689-697.

39. Piterman, N. From nondeterministic Büchi and Streett automata to deterministic parity automata. Log. Methods Comput. Sci. 2007, 3(3), 1-21.

40. Laroussinie, F.; Markey, N. Quantified CTL: Expressiveness and complexity. Log. Methods Comput. Sci. 2014, 10(4), 1-45.

41. Laroussinie, F.; Markey, N. Augmenting ATL with strategy contexts. Inf. Comput. 2015, 245, 98-123.

42. Bulling, N.; Dix, J.; Jamroga W. Model checking logics of strategic ability: Complexity. In Specification and Verification of Multi-Agent Systems; Springer: Berlin, Heidelberg, 2010; pp. 125-159.

43. Alur, R.; Henzinger, T.A.; Mang, F.Y.C.; Qadeer, S.; Rajamani, S.; Tasiran, S. MOCHA: Modularity in Model Checking. In Computer Aided Verification. In Proceedings of CAV, Vancouver, BC, Canada, 28 June-2 July 1998; pp. 521-525.

44. Lomuscio, A.; Qu, H.; Raimondi, F. MCMAS: An open-source model checker for the verification of multi-agent systems. Int. J. Softw. Tools Technol. Transf. 2017, 19, 9-30.

45. van der Hoek, W.; Wooldridge, M.J. Cooperation, knowledge, and time: Alternating-time temporal epistemic logic and its applications. Stud. Log. 2003, 75, 125-157.

46. Jamroga, W.; Ågotnes, T. What Agents Can Achieve Under Incomplete Information. In Proceedings of the AAMAS, Hakodate, Japan, 8-12 May 2006; pp. 232-234.

47. Belardinelli, F.; Lomuscio, A.; Malvone, V. An Abstraction-Based Method for Verifying Strategic Properties in Multi-Agent Systems with Imperfect Information. In Proceedings of the AAAI, Honolulu, HI, USA, 27 January-1 February 2019; pp. 6030-6037.

48. Xiong, L.; Guo, S. Model Checking Dynamic Strategy Logic with Memoryless Strategies. In Proceedings of the CSAE 2020, Sanya, China, 20-22 October 2020; pp. 68:1-68:5.

49. Jamroga, W.; Tabatabaei, M. Information Security as Strategic (In)effectivity. In Proceedings of the STM 2016, Heraklion, Greece, 126-27 September 2016; pp. 154-169.

50. Wu, K.; Fang, L.; Xiong, L.; Lai, Z.; Qiao, Y.; Rong, F. Automatic Synthesis of Generalized Winning Strategies of Impartial Combinatorial Games Using SMT Solvers. In Proceedings of the IJCAI, Yokohama, Japan, 7-15 January 2020; pp. $1703-1711$.

51. Belardinelli, F.; Condurache, R.; Dima, C.; Jamroga, W.; Knapik, M. Bisimulations for verifying strategic abilities with an application to the ThreeBallot voting protocol. Inf. Comput. 2021, 276, 104552. 